\title{
Musashi regulates follicle stem cell maintenance and epithelial niche homeostasis in the Drosophila ovary.
}

Nicole A. Siddall, Franca Casagranda ${ }^{1}$, Nicole Dominado ${ }^{1}$, James Heaney ${ }^{1}$, Jessie M. Sutherland ${ }^{2,3}$, Eileen A. McLaughlin ${ }^{2,3,4,5}$, Gary R. Hime ${ }^{1,6^{*}}$

1. Department of Anatomy and Neuroscience, The University of Melbourne, Parkville, Victoria, Australia, 3010

2. Priority Research Centre for Reproductive Science, Schools of Biomedical Science \& Pharmacy, University of Newcastle, Callaghan, Australia, 2308

3. Hunter Medical Research Institute, New Lambton Heights, Australia, 2305

4. School of Biological Sciences, University of Auckland, Auckland New Zealand 1010

5. School of Science, Western Sydney University, Penrith, NSW, 2751, Australia.

6. Centre for Stem Cell Systems, The University of Melbourne, Parkville, Victoria, Australia, 3010

Corresponding author:

Gary Hime

Department of Anatomy and Neuroscience, The University of Melbourne, Parkville, Victoria, Australia, 3010

Tel: +61-3-8344-5796

EM: g.hime@unimelb.edu.au

Keywords: Musashi, stem cell, Drosophila 


\begin{abstract}
The Musashi (Msi) family of RNA-binding proteins regulate maintenance and differentiation of stem cells in multiple tissues of different species. Here, we use the powerful system of the Drosophila ovary to uncover an intrinsic requirement for Msi in both the maintenance of the follicle stem cell population and regulation of the architecture of the follicle stem cell niche. Further, we demonstrate an associated G2 lag of cycling somatic cells within the niche when Msi function is abrogated. Additionally, we show that Msi interaction with the Wnt pathway influences differentiation of anterior germarial escort cells from cells within the follicle stem cell niche region. This provides further evidence that escort cells can be derived from follicle stem/precursor cells and that Msi regulates lineage differentiation of follicle stem cell daughters.
\end{abstract}




\section{Introduction}

Adult stem cells possess both the ability to self-renew and give rise to differentiated cells in order to maintain tissue homeostasis in multicellular organisms (Watt \& Hogan, 2000). For stem cells within a tissue to retain the capacity to maintain that tissue, self-renewal must occur through one of two mechanisms; either by asymmetrically dividing, or at the population level by population asymmetry (Simons \& Clevers, 2011). Most adult stem cells reside in specialized environments (niches) where they receive signals that regulate their behaviour (reviewed in (Stine \& Matunis, 2013)). The position of a stem cell within its microenvironment remains a critical determinant for the outcome of the stem cell, whether it be differentiation, division, or death. Additionally, stem cells in many tissue types have been shown to have the capacity to compete for niche occupancy under normal tissue homeostatic conditions to ensure healthy maintenance of stem cells and their niches (Amoyel, Simons, \& Bach, 2014; de Navascues et al., 2012; Klein \& Simons, 2011; Kronen, Schoenfelder, Klein, \& Nystul, 2014; Simons \& Clevers, 2011). This property of stem cells suggests that population asymmetry is a mechanism that governs stem cell maintenance to some extent in many niches (Stine \& Matunis, 2013).

In Drosophila melanogaster, the ovary provides an excellent model system for the study of stem cell behaviour. The Drosophila ovary is comprised of approximately $16-18$ individual ovarioles, which are sequential chains of egg chambers, with the most mature chambers found furthest from the anterior germarium (Spradling, 1993). The germarium, where germ cell development begins, is comprised of 3 regions (Fig. 1a). In region 1, germline stem cells (GSCs) reside within a niche of somatic cap cells (CC), terminal filament cells (TF) and escort cells (ECs), and divide asymmetrically to produce daughter cystoblasts. The cystoblast 

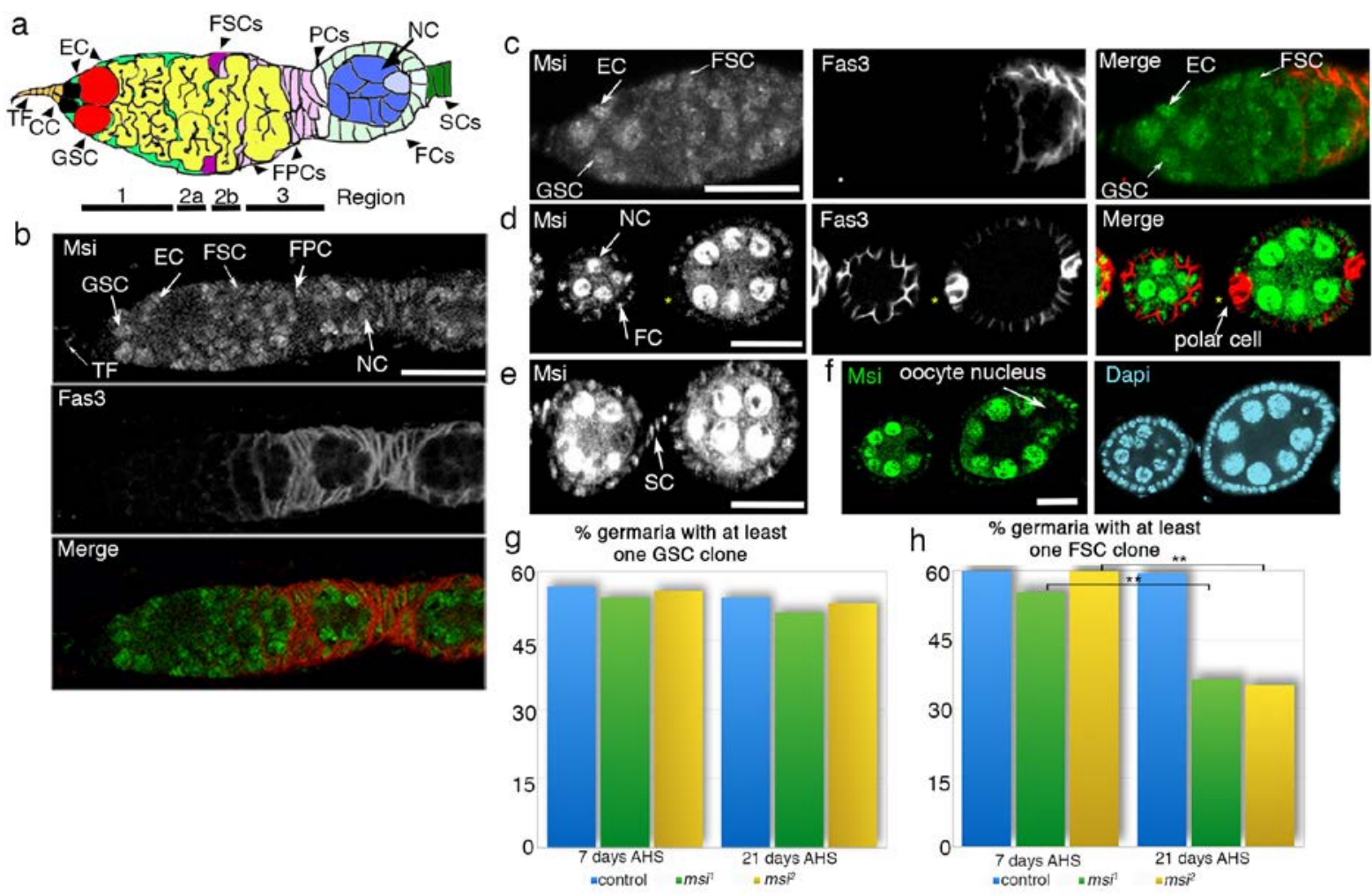

Figure 1. Musashi protein expression in the Drosophila ovary and it's requirement in follicle stem cell maintenance. (a) Cartoon depiction of the early stages of Drosophila ovary development showing the cell types, including terminal filament cells and cap cells (TF; CC, orange), escort cells (EC, green), germline stem cells (GSC, red with black nuclei attached to the cap cells), germ cells (yellow), follicle stem cells (FSCs, dark purple), follicle precursor cells (FPCs, light purple), polar cells (PCs, white), nurse cells (NC, dark blue), follicle cells (FCs, light green) and stalk cells (SCs, dark green). (b-f) Confocal micrographs of Msi expression in wildtype ovaries. (b-c) In the anterior of the germarium, Msi expression is observed in GSCs, ECs and in somatic terminal filament (TF) and cap cells (CC). Msi expression is also observed in all somatic cells within the FSC niche region, including FSCs and FPCs. A reduction of visible Msi expression in 4-8 cell germline cysts was consistently observed. (d-f) In later stage follicles, Msi expression was observed in nurse cells, mature follicle cells and stalk cells. ${ }^{*}$ in $d$ denotes the polar cells, where Msi expression was not detectably by immunofluorescence. (g) The percentage of germaria containing at least on negatively marked control (blue), $\mathrm{msi}^{1}$ (green) or $\mathrm{msi}^{2}$ (yellow) GSC clone generated by Flp-FRT mediated recombination at 7 days $\mathrm{PCl}$ (56.7\%, $\mathrm{N}=97 ; 54.16 \%, \mathrm{~N}=96$ and 55.67\%, $\mathrm{N}=97$ respectively) and 21 days $\mathrm{PCl}(54.16 \%, \mathrm{~N}=96$; $50.98 \%, \mathrm{~N}=102$ and 53\%, N=100 respectively). No significant differences were observed between genotypes as analysed by Chi-square analysis. (h) The percentage of germaria containing at least on negatively marked control (blue), $\mathrm{si}^{1}$ (green) or $m \mathrm{si}^{2}$ (yellow) FSC clone generated by Flp-FRT mediated recombination 7 and 21 days $\mathrm{PCl}$. A significant reduction in the frequency of $m s^{1}(36.27 \%, \mathrm{~N}=102$; Chi-square $\mathrm{p}=.0075)$ and $m \mathrm{si}^{2}(35 \%, \mathrm{~N}=100$; Chi-square $\mathrm{p}=.0055$ ) mutant FSC clones at 21 days $\mathrm{PCl}$ was observed. Scale bars, $20 \mu \mathrm{m}$. 
continues to divide, with the end result being the production of 16 -cell cysts in region $2 \mathrm{~b}$ of the germarium (Spradling, 1993). Somatic ECs are typically quiescent and support the developing germline, with tight association of ECs with the germline being necessary for germline development (Banisch, Maimon, Dadosh, \& Gilboa, 2017; Kirilly, Wang, \& Xie, 2011; Maimon, Popliker, \& Gilboa, 2014; Yang et al., 2017). ECs are highly dynamic in nature and protrude within the packed germarium to invade and engulf the germline (Banisch et al., 2017). ECs also differ in their shape, size and ability to associate with germ cells depending on their position within the germarium, suggesting that ECs are functionally diverse (Banisch et al., 2017).

About one third of the way down the germarium, germline cysts exchange their escort cell covering for the daughters of follicle stem cells (FSCs), which reside in region $2 a / 2 b$ of the germarium (Margolis \& Spradling, 1995). Posterior ECs can contact FSCs and may act as a cellular niche, along with the basement membrane and more anterior cap cells, which can signal to the distant FSCs (Banisch et al., 2017; O'Reilly, Lee, \& Simon, 2008; Song \& Xie, 2003; Waghmare \& Page-McCaw, 2018; X. Wang \& Page-McCaw, 2014). FSC and follicle precursor cell fate specification and differentiation are dependent upon several signalling pathways. Notch activity in the FSC region $(2 \mathrm{a} / 2 \mathrm{~b})$ is required for lateral migration of FSCs across the germarium in either an anterior or posterior manner (Nystul \& Spradling, 2010) and for polar cell (PC) specification (Lopez-Schier \& St Johnston, 2001). Wingless (Wg) signalling is necessary for correct FSC differentiation and specification. Wg signalling produced from distant cap cells has been shown to be essential for FSC proliferation and survival, and upregulation or loss of Wg signalling has been shown to result in the loss of FSCs (Song \& Xie, 2003; X. Wang \& Page-McCaw, 2014). FSC specification has also been shown to be dependent 
upon the levels of Hedgehog $(\mathrm{Hh})$ and $\underline{J}$ Anus Kinase protein and the $\underline{\text { Signal }}$ Transducer and Activator of Iranscription (JAK-STAT) signals received (Dai, Peterson, Kenney, Burrous, \& Montell, 2017; Vied, Reilein, Field, \& Kalderon, 2012; Zhang \& Kalderon, 2001).

The exact number of FSCs within the germarium remains a controversial topic. Early studies proposed that 2-3 somatic FSCs divide to give rise to a daughter stem cell and follicle precursor cell (FPCs). FPCs then differentiate into posterior follicle cell (FC) types, including polar, stalk and main body epithelial cells (Margolis \& Spradling, 1995). A more recent study, which utilised a novel clonal marking system, proposed that a population of approximately 14 FSCs exist in the germarium, located in three layers within region $2 a / 2 b$, typically considered the FSC niche region, where posterior ECs interact with FSCs (Reilein et al., 2017). Another report has challenged this and suggest that there are likely to be 1-4 FSCs positioned at the $2 \mathrm{a} / 2 \mathrm{~b}$ boundary region of the germarium, with 2 FSCs per ovariole active (Fadiga \& Nystul, 2019) (Fig. 1a).

Regardless of how many FSCs reside in the ovary, evidence from several studies suggest that somatic cells within region $2 \mathrm{a} / 2 \mathrm{~b}$ of the germarium can move laterally, anteriorly or posteriorly across the germarium. These include cross-migrating FSCs (Nystul \& Spradling, 2007, 2010), anterior movement of FSCs as observed by live cell imaging (Reilein et al., 2017), or movement of posterior escort cells through the germarium. This capacity to migrate gives stem cells opportunity to compete for niche occupancy and enables stem cell replacement and tissue homeostasis. Therefore, identifying factors regulating FSCs and other epithelial cells within region $2 \mathrm{a} / 2 \mathrm{~b}$ of the germarium remains critical to gaining clarity into the 
mechanisms governing epithelial stem cell maintenance and differentiation, and niche homeostasis.

Recent studies report the importance of RNA-binding proteins (RBPs) as essential regulators of epithelial stem cell maintenance and tissue homeostasis in many different organ systems. RBPs mediate post-transcriptional regulation and their interaction with mRNAs result in the formation of complex genetic networks. In mice, the vertebrate Musashi (Msi) RBPs, Msi-1 and Msi-2, have been shown to have an important requirement in intestinal epithelial tissue regeneration after injury, despite being dispensable for normal tissue homeostasis (Yousefi et al., 2016). Here, we report on the effects of loss of Drosophila Msi from epithelial stem cells and somatic cells within the FSC niche region of the Drosophila germarium.

We previously demonstrated that Drosophila Msi is required for the maintenance of germline stem cells (GSCs) in the testis (Siddall, McLaughlin, Marriner, \& Hime, 2006). Its vertebrate orthologues, Msi-1 and Msi-2, have both been described as having important functions in the regulation, proliferation and maintenance of neural, gastrointestinal, mammary, stomach and hematopoietic stem cells (Asai, Okano, \& Yasugi, 2005; Ito et al., 2010; Kaneko et al., 2000; Kharas et al., 2010; Park et al., 2014; Potten et al., 2003; Sakakibara et al., 2002; Y. Wang, Jiang, \& Fan, 2015) and play key roles in vertebrate spermatogenesis (Sutherland et al., 2014; Sutherland, Sobinoff, Fraser, et al., 2015) and folliculogenesis (Sutherland, Sobinoff, Gunter, et al., 2015). Here, we propose that Drosophila Msi is required for FSC maintenance and plays an important role in FSC niche homeostasis, maintaining the differentiation potential of somatic cells within the FSC niche region. We show that loss of Msi in FSCs by Flp-mediated FRT clonal analysis leads to a gradual loss of FSCs. An increase in the number of cross- 
migrating Notch receptive cells anterior to region $2 \mathrm{a} / \mathrm{b}$, coupled with lineage tracing analysis, show that loss of Msi from within the FSC niche region results in an increase in the number of anterior ECs. This phenotype is enhanced when Wnt signalling activity is increased by loss of Axin (Axn), and we observed an increase in Wnt reporter activity when Msi function was lost. We propose that Msi is necessary for epithelial niche homeostasis by contributing to the regulation of Wnt pathway activity within the FSC niche region. 


\section{Results}

We previously demonstrated an intrinsic requirement for Msi in male GSCs to maintain GSC identity (Siddall et al., 2006). Our first aim was to investigate whether there is a similar requirement for Msi in GSC or FSC maintenance in the ovary. Analysis of Msi protein distribution in the ovary using an antibody specific to Msi (Hirota et al., 1999; Siddall et al., 2006) showed Msi expression in ovarian germline and somatic cells (Fig. 1b-f). Germarial germline expression of Msi was observed in GSCs and differentiated germ cells (Fig. 1b-c). Interestingly, a reduction of Msi protein expression levels was consistently observed by immunofluorescence in early 2-4 cell cysts within the germarium (Fig. 1b-c). Msi expression persisted in nurse cells in later stage egg chambers but was absent from the oocyte (Fig. 1df). In somatic cells, Msi expression was observed in TF, EC and FSCs (Fig. 1b), differentiated FCs and stalk cells (Fig. 1d-e), but was not detectable by immunofluorescence in polar cells (Fig. 1d). FSCs were identified as somatic cells located at the region $2 a / 2 b$ junction, where the membranes of cells are labelled with low levels of Fasciclin 3 (Fas3) (Fig. 1b-c) (Kirilly, Spana, Perrimon, Padgett, \& Xie, 2005). Fas3 expression is maintained at high levels in polar cells despite being reduced in mature epithelial cells, making it a suitable polar cell marker (Fig. 1d).

To determine if Msi is required for either germline, or somatic stem cell maintenance, we generated Flp-mediated FRT (FIp-FRT) msi loss of function GSC clones and FSC clones marked by the absence of GFP, utilising two different $m s i$ mutant alleles, $m \mathrm{si}^{1}$ (a reported null mutant) and $m \mathrm{si}^{2}$ (a reported hypomorphic allele, (Nakamura, Okano, Blendy, \& Montell, 1994)) and measured whether clones were maintained over time. At 7 days post clone induction ( $\mathrm{PCl})$, control GSC clones were observed at a similar frequency $(56.7 \%, \mathrm{~N}=97)$ to $m \mathrm{si}^{1}(54.16 \%, \mathrm{~N}=96)$ 
and $m s^{2}(55.67 \%, \mathrm{~N}=97)$ mutant clones (Fig. $\left.1 \mathrm{~g}\right)$. At 21 days $\mathrm{PCl}$, no significant reduction in the frequency of control $(54.16 \%, \mathrm{~N}=97), \mathrm{msi}^{1}(50.98 \%, \mathrm{~N}=102)$ or $m \mathrm{si}^{2}(53.06, \mathrm{~N}=100)$ clones was observed (Fig. 1g). These findings were unexpected given the high level of Msi antibody expression observed in the GSCs of the adult ovary (Fig. 1b-c) and the requirement for Msi in maintenance of spermatogonial stem cells (Siddall et al., 2006) .

We next sought to answer whether Msi may play a role in the regulation of FSC differentiation, given its observed expression in FSCs in the adult germarium (Fig. 1b-c). Accordingly, we assayed for the frequency of control and msi mutant FSC clones at the $2 a / 2 b$ junction at 7 days and 21 days $\mathrm{PCl}$. At 7 days $\mathrm{PCl}$, control $\mathrm{FSC}$ clones were observed at a frequency of $59.79 \%$ ( $N=97$; Fig. $1 \mathrm{~h}$ ) which was not significantly different to the frequency of $m s^{1}$ mutant FSC clones $(55.21 \%, \mathrm{~N}=96)$ or $m s^{2}$ mutant FSC clones $(59.79 \%, \mathrm{~N}=97)$ observed at the same timepoint. At 21 days $\mathrm{PCl}$, the frequency of control FSC clones remained stable $(59.38 \%, N=96)$. However, we observed a significant reduction in the frequency of $m s i^{1}$ (36.27\%, $\mathrm{N}=102$; Chi-square $\mathrm{p}=.0075)$ and $m \mathrm{si}^{2}(35 \%, \mathrm{~N}=100$; Chi-square $\mathrm{p}=.0055)$ mutant FSC clones at 21 days $\mathrm{PCl}$ (Fig. 1h), indicating a requirement for Msi in FSC maintenance.

Homozygous msi mutants are viable into early adulthood, albeit they are less fit than their heterozygous and wild-type counterparts and rarely survive for more than a few days. To further explore the function of Msi in ovary morphogenesis, adult ovaries were dissected from $m s i^{1 / 1}$ null and $m s i^{1 / 2}$ transheterozygote mutant females 2-3 days post eclosion. Germaria dissected from msi mutants were swollen and appeared to contain excess Vasalabelled germ cells in region 1-2b (Fig. 2a-b). Using the germ-cell marker Vasa coupled with the fusome marker 1B1 to identify germline cysts, we counted significantly more germline 


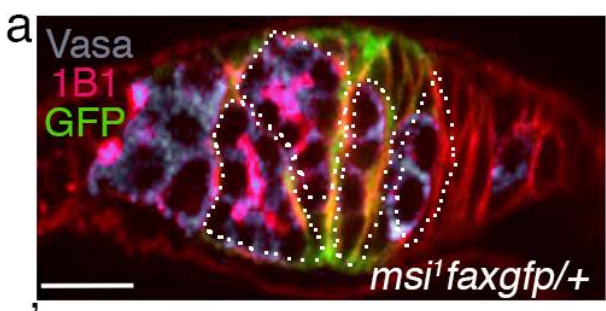

$a^{\prime}$

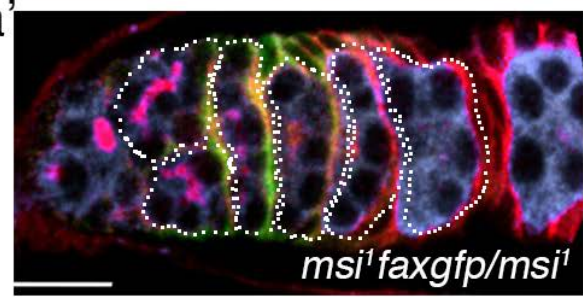

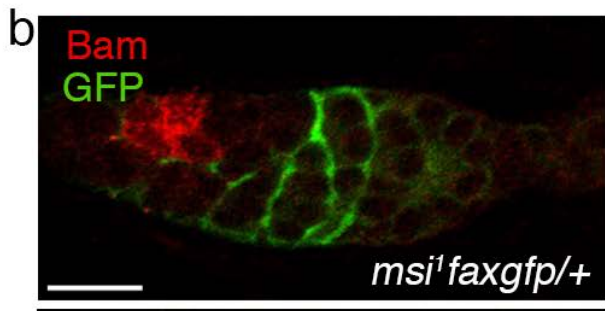

$b$

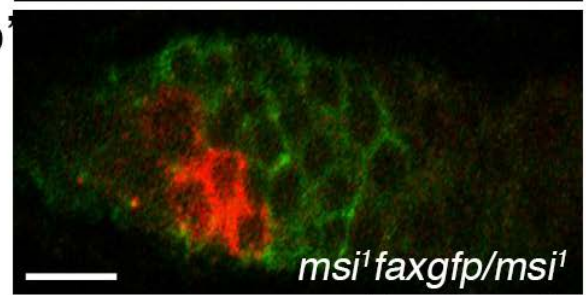

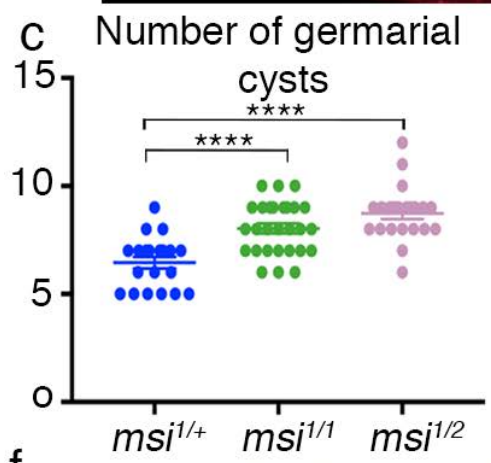

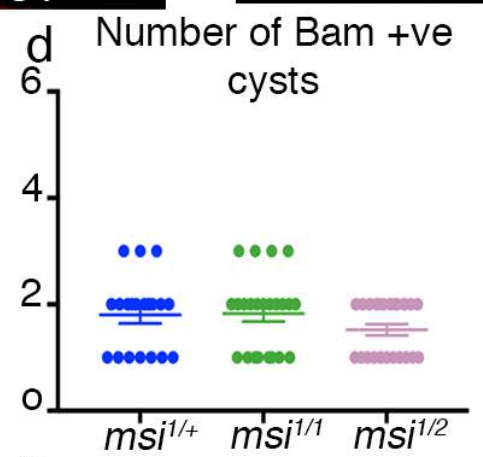

e Number of region 2-3
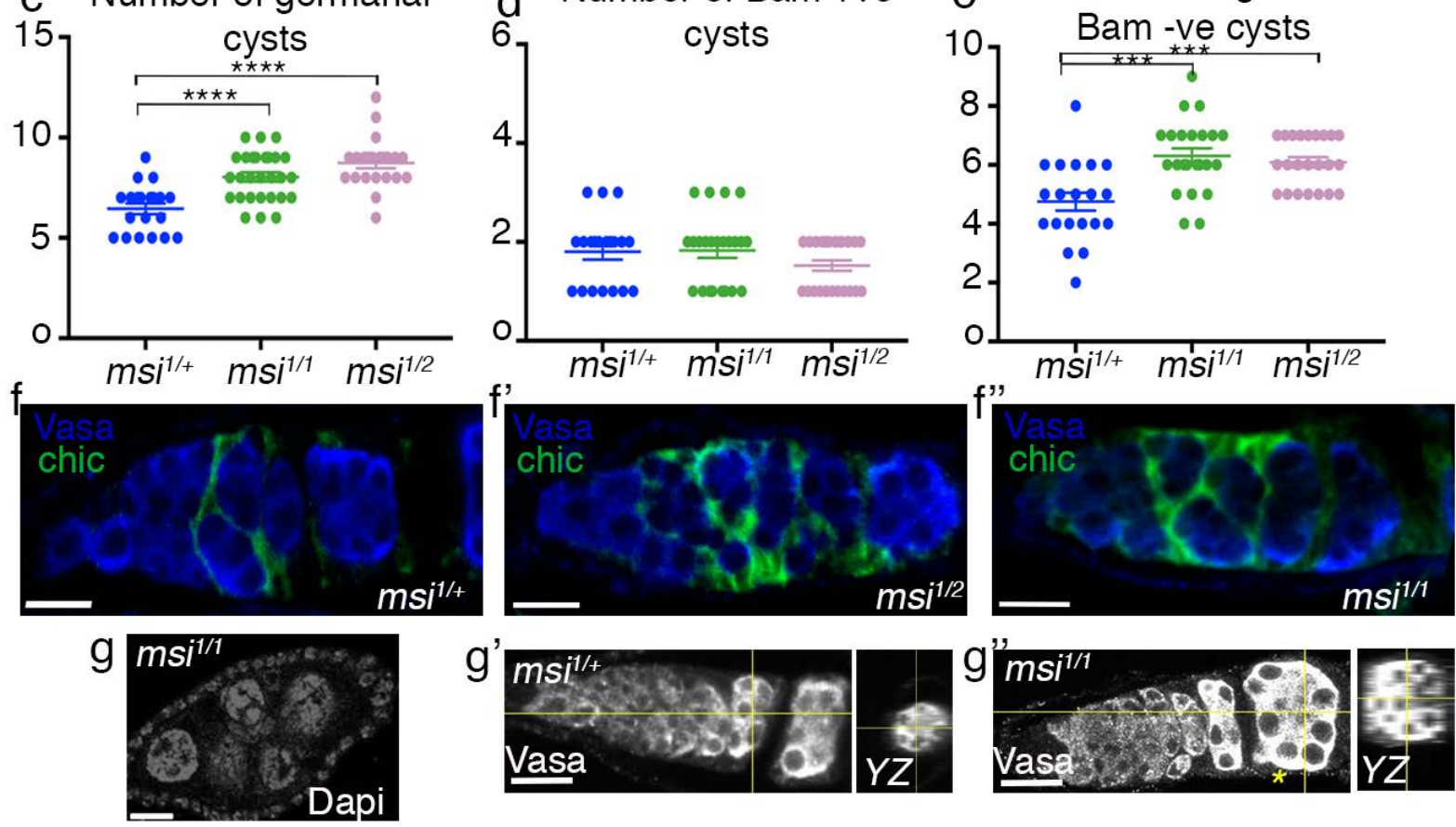

Figure 2. msi mutations cause an increase in the number of germline cysts in the FSC niche region (region $2 a / 2 b$ ), an expansion of the FSC niche and follicle budding defects. (a-b) Confocal micrographs of control (a and b) and msi null ( $a$ ' and b') germaria. ( $a$ and $a^{\prime}$ ) Germline cysts (dotted outlines) were counted in germaria labelled with Vasa (germ cells), 1B1 (fusomes showing connections between germ cells), and fax::GFP, a marker of escort cell cytoplasm. (c) Scatterplot of the average number of germline cysts in regions 1 to 3 of germaria ( \pm SEM) from heterozygote control (blue; 6.45 \pm .27; $\mathrm{N}=20$ ), $\mathrm{msi}^{1 / 1}$ (green; 8.036 $\pm .227 ; \mathrm{N}=28$ ) and transheterozygote $m s^{1 / 2}$ flies (pink; 8.727 $\pm .265 ; \mathrm{N}=22$ ). Welch's two-tailed t-tests reveal a significant increase in the average number of cysts in both msi null and transheterozygote mutants compared to control germaria $(p<0.0001$ in both cases). In b and b', germaria were labelled with Bag of Marbles (Bam) and fax::GFP. (d) Graph of the average $\left( \pm \mathrm{SEM}\right.$ ) number of Bam positive germline cysts in heterozygote control (blue; $1.8 \pm .16 ; \mathrm{N}=20$ ), $\mathrm{msi}^{1 / 1}$ (green; $1.82 \pm .149 ; \mathrm{N}=23$ ) and transheterozygote $\mathrm{msi}^{1 / 2}$ flies (pink; $1.52 \pm .11 ; \mathrm{N}=23$ ). No significant difference between the genotypes was observed. (e) Graph of the average ( \pm SEM) number of Bam negative germline in region 2-3 of the germarium (e) in heterozygote control (blue; $4.75 \pm .31 ; \mathrm{N}=20$ ), $\mathrm{msi}^{1 / 1}$ (green; 6.304 $\pm .255 ; \mathrm{N}=23$ ) and transheterozygote $\mathrm{msi}^{1 / 2}$ flies (pink; 6.087 $\pm .177 ; \mathrm{N}=23$ ). Welch's two-tailed t-tests show a significant increase in the number of germline cysts posterior to the region of Bam expression in both $m s^{1 / 1}(p=.0004)$ and $m s i^{1 / 2}$ $(p=.0007)$ germaria compared to control. ( $\left.f-f^{\prime \prime}\right)$ Chickadee expression (green) and Vasa (blue) in heterozygote control (f), $m s i^{1 / 2}\left(f^{\prime}\right)$ and $m s i^{1 / 1}\left(f^{\prime \prime}\right)$ germaria. (g) $m s^{1 / 1}$ egg chamber with less than the correct complement of nurse cells. $\left(g^{\prime}-g^{\prime \prime}\right) X Y$ and $Y Z$ projections of control $\left(g^{\prime}\right)$ and $m s^{1 / 1}$ mutant $\left(g^{\prime \prime}\right)$ germaria. In $g^{\prime \prime}$, a stage 2 cyst $\left(\right.$ yellow ${ }^{*}$ ) has more than the correct complement of germ cells. Scale bars, $10 \mu \mathrm{m}$. 
cysts in msi mutant germaria compared to control germaria (Fig. 2c). Furthermore, these excess cysts were observed in regions $2 \mathrm{a} / \mathrm{b}$ and 3 , posterior to the Bag-of-Marbles (Bam) antibody expression domain, which labels 2-4 cell gemline cysts (Ohlstein \& McKearin, 1997) (Fig. 2d-e). In these regions, we observed an average of $4.75 \pm .31(\mathrm{~N}=20)$ germline cysts in control germaria, compared to $6.304 \pm .255(\mathrm{~N}=23)$ and $6.087 \pm .177(\mathrm{~N}=23)$ in $m s i^{1 / 1}$ and $m s^{1 / 2}$ mutants respectively (Fig. 2e).

A previous study identified Chickadee (Chic), the vertebrate homolog of Profilin, as a marker of the FSC niche region, since expression is reportedly upregulated specifically in a narrow strip of somatic cells at the regions $2 \mathrm{a} / 2 \mathrm{~b}$ boundary (Huang et al., 2014) (Fig. $2 \mathrm{f}$ ). $m s i^{1 / 1}$ and $m s i^{1 / 2}$ mutants both exhibited an extended domain of Chic expression in region $2 \mathrm{a} / \mathrm{b}$, the region where excess germarial cysts were observed (Fig. 2f'-f"). Consistent with defects in FSC maintenance and FPC differentiation, approximately $10 \%$ of homozygous $\mathrm{msi}^{1 / 1}$ mutants $(\mathrm{N}=220)$ exhibited packaging defects, with germline egg chambers containing either less or more than the correct complement of NCs (Fig. 2g-g"). Taken together, our clonal data and phenotypic analysis suggest a requirement for Msi in FSC maintenance and differentiation of pre-follicle cells within the FSC niche region.

Clonal data using Flp-FRT mediated recombination revealed a slow loss of msi mutant FSCs from the niche between 7 and 21 days $\mathrm{PCl}$ (Fig. 1h). Surprisingly, we observed a number of negatively marked somatic cell clones in positions anterior to region $2 \mathrm{a} / 2 \mathrm{~b}$ of the germarium, which appeared to be EC clones expressing the somatic cell marker Traffic Jam (Tj) (Fig. 3b). These clones were generated under both control conditions and with msi mutant alleles. The percentage of germaria containing at least one control EC clone in the absence of any FSC 


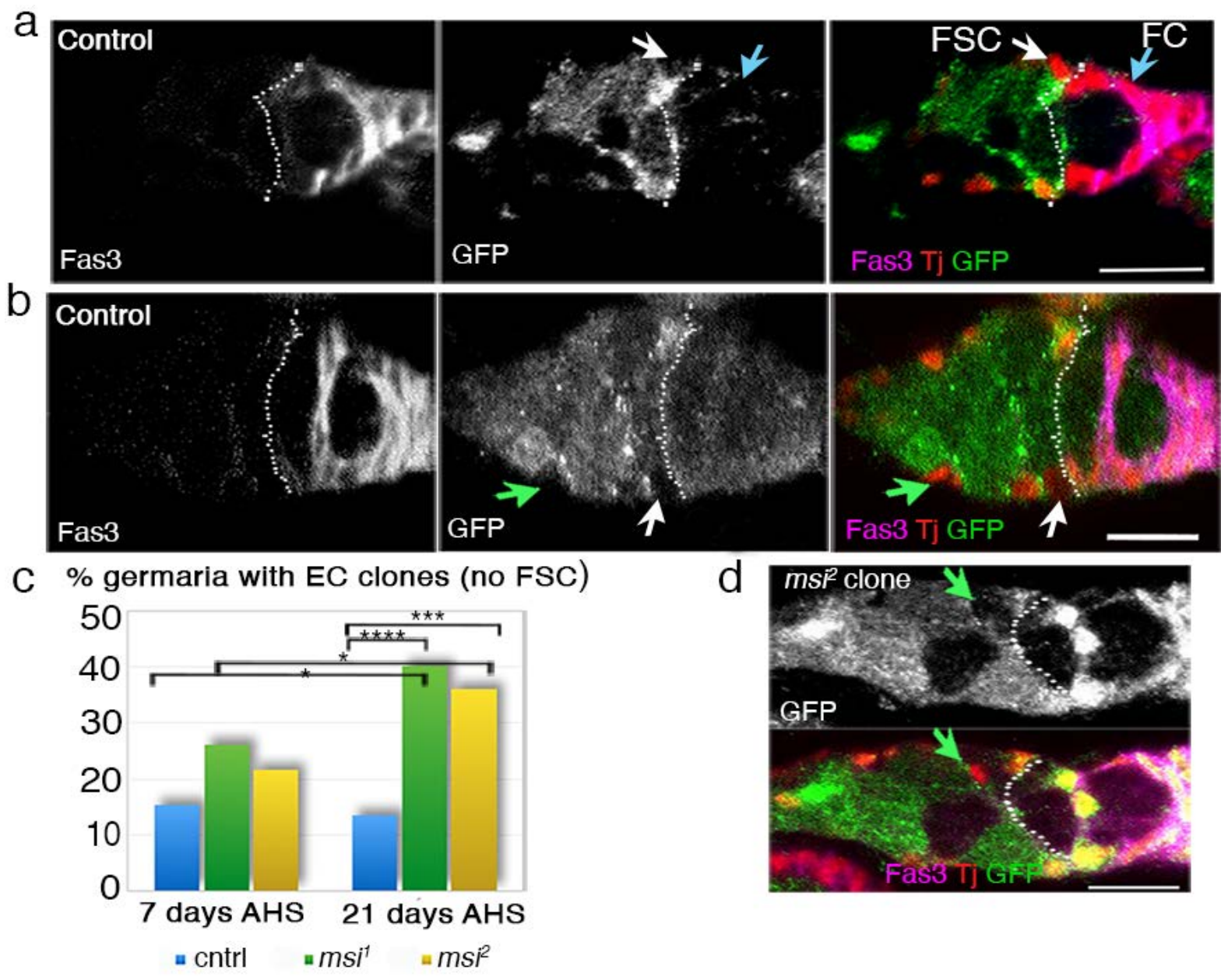

Figure 3. Loss of Msi function causes an increase in the production of anterior EC clones. (ab) Single plane, confocal micrographs showing Traffic Jam ( $\mathrm{Tj}$, red) positive, GFP-negative clones generated by FIp-FRT. A FSC clone (white arrow), FC clone (blue arrow) and EC clone (green arrow) are depicted. (c) Column graph of the percentage of germaria containing at least one wild-type control EC clone (blue), $m s^{1}{ }^{1} \mathrm{EC}$ clone (green) or $m \mathrm{si}^{2} \mathrm{EC}$ clone (yellow) at 7 days and 21 days after heat shock (AHS). Chi-square analysis revealed an increase in the \% of germaria containing either a $m s^{1}$ or $m s^{2}$ EC clone in the absence of any FSC/FPC clones between the two timepoints ( $m \mathrm{si}^{1}$ : Chi-square $\mathrm{p}=.03$; $m \mathrm{si}^{2}$ : Chi-square $\left.\mathrm{p}=.026\right)$. The $\%$ of germaria containing control EC clones observed in the absence of any FSC clones remained constant between 7 days $\mathrm{PCl}(15.46 \%)$ and 21 days $\mathrm{PCl}(13.54 \%)$. At three weeks $\mathrm{PCl}$, significantly more germaria exhibited both $\mathrm{msi}^{1}(40.19 \%$; Chi-square $\mathrm{p}<.0001)$ and $m \mathrm{si}^{2}(36 \%$; Chi-square $p=.003$ ) anterior EC clones compared to control EC clones (13.54\%). (d) A single plane confocal micrograph depicting a single TJ positive (green arrow) $m \mathrm{si}^{2} \mathrm{EC}$ clone centrally in the germaria just anterior to region $2 a / 2 b$. White dotted lines represent the region $2 a / 2 b$ boundary. Scale bars, $10 \mu \mathrm{m}$. 
clones remained constant between 7 days $\mathrm{PCl}(15.46 \%)$ and 21 days $\mathrm{PCl}(13.54 \%)$. In contrast, the percentage of germaria containing either $m s^{1}{ }^{1}$ or $m s^{2}$ EC clones in the absence of any FSC or FPC clones significantly increased between the two timepoints (msi ${ }^{1}$ : Chi-square $p=.03$; $m s^{2}$ : Chi-square $\mathrm{p}=.026$ ) (Fig. 3c). Strikingly, by three weeks $\mathrm{PCl}$, there were significantly more germaria exhibiting both $m s i^{1}(40.19 \%$; Chi-square $p<.0001)$ or $m s i^{2}(36 \%$; Chi-square $p=.003)$ anterior EC clones than control EC clones (13.54\%). EC clones were observed in both region 1 of the germaria (Fig. 3b), and in a central location within the germaria just anterior to region 2a/2b (Fig. 3d). Some of these clones were basally located and appeared very close to the outer surface of the germaria, indicative of morphological and differentiation defects.

Our results raised the question as to whether the increase in msi mutant EC clone generation is due to an increase in EC division or aberrant differentiation of FSCS/FPCs, leading to an anterior shift in differentiation of FSCs and/or FPCs from the niche. A previous study has reported that differentiation defective FSCs can be displaced in the germarium, with Mosaic Analysis with a Repressible Cell Marker (MARCM) revealing that these cells commonly display aberrant, axon like projections that fail to extend along the circumference of the germaria (Hartman et al., 2015). This was in contrast to control FSCs, which were shown to extend a thick, axon-like projection across the circumference of the germarium along the border of regions $2 a$ and $2 b$ (Hartman et al., 2015) (Supp. Fig. 1). We generated GFP-labelled $m s^{1}$ mutant FSC clones by MARCM and used a membrane bound GFP (CD8::GFP) to better assess the morphological characteristics of mutant cells by confocal microscopy at 3 weeks post clone generation. Many GFP-positive $m s i^{1}$ mutant clones within the FSC niche were located close to the outer surface of the germarium. These clones often appeared as single clones without progeny and displayed aberrant, shortened thick projections that did not extend 
along the circumference of the germarium (Supp. Fig. 1). Interestingly, both msi and control GFP-positive EC clones were observed in germaria at 3 weeks after clone induction (Supp Fig. 1). Only $3.3 \%$ of germaria $(\mathrm{N}=30)$ contained a marked control EC clone in the absence of a FSC clone, while $20 \%$ of germaria contained a marked $m s^{1}$ EC clone in the absence of a FSC clone (Supp. Fig. 1). In 3 of these $7 \mathrm{msi}^{1}$ mutant EC clones, we observed axon like projections extending from the FSC niche region to the clone anterior or region 2a. Taken together, our MARCM clonal and Flp-FRT analysis strongly suggest that loss of Msi from FSC clones results in delayed or defective differentiation and a shift in FSCs and/or their daughters towards an anterior fate in the germarium.

To gain further insight into the function of Msi in the FSC niche region, we used the ReDDM (Repressible Dual Differential stability cell Marker) lineage tracing system coupled with a FSC niche specific $10930-$ Gal4 driver (10930-Gal4 ${ }^{\text {ReDDM }}$ ) and a UAS-msi ${ }^{R N A i}$ transgene (Supp. Fig. 2) to knockdown Msi in the niche and trace the progeny cells derived from this region. ReDDM enables the temporal-spatial visualisation of cells in the Gal4 domain by using a Gal4 responsive, membrane-bound GFP transgene (mCD8-GFP) with a short half-life, whilst tracing the differentiated progeny using a H2B-RFP transgene with a long half-life (Antonello, Reiff, Ballesta-Illan, \& Dominguez, 2015). 10930-Gal4 reportedly drives expression in region 2a/2b of the germaria where FSCs and their immediate daughters reside (Hartman et al., 2015; Hartman et al., 2010) and has previously been utilised to generate MARCM FSC clones (Hartman et al., 2015). While this driver predominantly restricts expression to the FSC niche in adult ovaries, expression of the driver has been reported in larval EC cells and occasional adult EC cells (Hartman et al., 2015; Sahai-Hernandez \& Nystul, 2013). The use of tub-Gal80 ${ }^{\text {ts }}$ ensured repression of the $10930-$ Gal4 $4^{\text {ReDDM }}$ system until 1-2 day old adult flies were shifted to 
$29^{\circ}$ for 2 weeks, enabling the restriction of tracing progeny from cells within the adult FSC niche. We observed an average of $5.095( \pm 0.3774, \mathrm{~N}=21)$ marked H2B::RFP cells anterior to region $2 a$ in control germaria (Fig. $4 a, c)$, suggesting that cells within the niche region can indeed give rise to escort cells, as previously reported (Reilein et al., 2017). Interestingly, knockdown of Msi within the niche region by RNAi using the 10930-Gal4 driver resulted in a significant increase in the number of H2B-RFP marked somatic cells $(9.762 \pm 0.4776, \mathrm{~N}=21)$ anterior to region $2 a / 2 b$ (Fig. $4 b$-c; t-test $p<.0001$ ). These results support the conclusion that FSC and/or FPC differentiation is skewed, with more cells taking on an anterior fate upon loss of Msi function from the FSC niche region.

Previous studies have shown that FSC daughters are able to migrate laterally across region $2 a / 2 b$ of the germarium, and on occasion can replace the opposite stem cell (Nystul \& Spradling, 2007, 2010). Additionally, FSC daughters reportedly possess an alternate anterior or posterior positional bias occurring at approximately equal frequencies, and the patterns of cross migration have been shown to be dependent upon Notch function (Nystul \& Spradling, 2010). A Notch signalling reporter (Gbe-Su(H) LacZ) has previously been shown to mark FSCs and their cross-migrating daughters in region $2 a / 2 b$ of the germarium (Nystul \& Spradling, 2010). In this study, we utilised an alternative Notch activity reporter, the Notch responsive element (NRE) driving GFP expression (Housden, Millen, \& Bray, 2012), to determine whether loss of Msi affects the positional bias of undifferentiated somatic cells within region $2 a / 2 b$ of the germarium. NRE-GFP has been shown to be expressed in a subset of cells in the $2 a / 2 b$ region in addition to polar cells and cap cells (Dai et al., 2017; Johnston, Bar-Cohen, Paroush, \& Nystul, 2016) (Fig 4d). In control $\left(m s^{1 /+}\right)$ germaria, we observed an average of $1.818 \pm .243$ (SEM) NRE-GFP expressing cells ( $\mathrm{N}=22$; Fig. 4h). This average was significantly higher in $\mathrm{msi}^{1 / 2}$ 


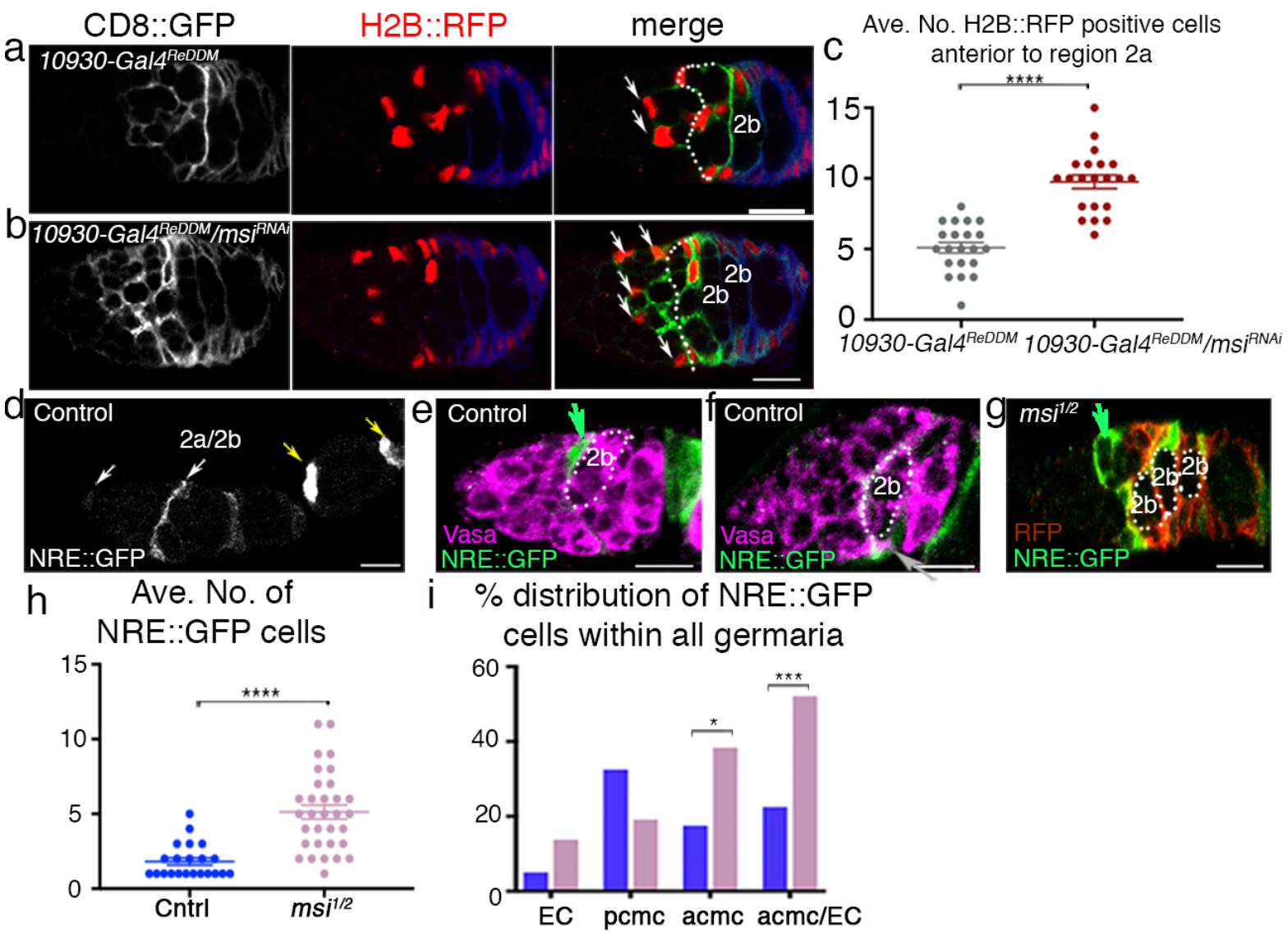

Figure 4. Loss of Msi function from the FSC niche results in an increase in the differentiation of anterior ECs. (a-b) Representative confocal micrographs of the ReDDM lineage tracing system from control (10930-Gal4 $\left.{ }^{R E D D M}\right)$ and $10930-G a l 4^{R E D D M} / \mathrm{msi}^{\text {RNAi }}$ mutant germaria. The FSC niche driver 10930-Gal4 drives a CD8::GFP transgene in the FSC niche region. The long lived Histone2B-RFP (H2B::RFP, red cells) allows for tracing of differentiated progeny derived from the cells within the region marked by 10930-Gal4,CD8::GFP. RFP-positive cells (white arrows) anterior to the region 2 a boundary (dotted line) were counted from (a) 10930Gal4 ${ }^{R E D D M}$ and (b) 10930-Gal4 ${ }^{R E D D M} / \mathrm{msi}^{\text {RNAi }}$ mutant germaria to generate the scatterplot in (c). Region $2 b$ cysts are labelled (2b). (c) Scatterplot depicting the average ( \pm SEM) number of H2B::RFP cells anterior to region 2a from 10930-Gal4 ${ }^{R E D D M}$ and $10930-$ Gal4 ${ }^{\text {REDDM }} / \mathrm{msi}^{\text {RNAi }}$ germaria. 5.095 0.3774 ( $\mathrm{N}=21) \mathrm{H} 2 \mathrm{~B}:$ :RFP cells were observed in control germaria, and 9.762 $\pm 0.4776(\mathrm{~N}=21)$ in $10930-\mathrm{Gal}^{R E D D M} / \mathrm{msi}^{R N A i}$ germaria (t-test $\left.\mathrm{p}<.0001\right)$. (d) NRE::GFP expression in a wild-type ovary. White arrows point to Notch activity in cap cells and in cells in region $2 \mathrm{a} / 2 \mathrm{~b}$ of the germaria. Yellow arrows point to polar cells. (e-g) Representative confocal micrographs of a NRE::GFP positive anterior cross migrating cell (green arrow, e) and posterior cross migrating cell (grey arrow $\mathrm{f}$ ) in control germaria and an escort cell in a $m s^{1 / 2}$ germarium (green arrow, g). Region $2 \mathrm{~b}$ cysts are outlined (white dots). (h) Scatterplot of the average number ( \pm SEM) of NRE::GFP positive cells from region 1 to region 3 of control $\mathrm{msi}^{1 /+}$

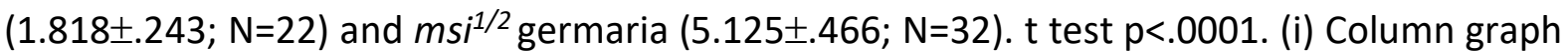
representing the $\%$ of total distribution of NRE::GFP positive cell types in $\mathrm{msi}^{1 /+}(\mathrm{Cntrl})$ and $m s i^{1 / 2}$ germaria. Chi-square test $(p=.0007)$ revealed both a highly significant increase in the frequency of anteriorly positioned NRE::GFP positive cmcs and escort cells combined in $\mathrm{msi}^{1 / 2}$ transheterozygotes (52.1\%), up from $22.5 \%$ in $\mathrm{msi}^{1 /+}$ controls, and a significant increase in NRE::GFP labelled acmcs in $\mathrm{msi}^{1 / 2}$ transheterozygotes (38.32\%), up from $17.5 \%$ in control germaria (Chi-square $p=.013$ ). Scale bars, $10 \mu \mathrm{m}$. 
transheterozygotes (5.125 $\pm .466 ; \mathrm{N}=32$; t-test $\mathrm{p}<.0001)$ (Fig. 4h). Additionally, we quantified the occurrences of NRE-GFP positive anteriorly positioned cross-migrating cells (acmcs; Fig. 4e), posteriorly positioned cmcs (pcmcs; Fig. 4f) and ECs (Fig. 4g). These cells were determined by their location within the germarium. Acmcs are located along the anterior face of the $2 b$ cyst within germarium, but not in the previously defined position of a FSC (Nystul \& Spradling, 2007), and pcmcs are located along the posterior face of the $2 \mathrm{~b}$ cyst. Escort cells were defined as cells anterior to 2 a cysts. Loss of Msi caused a significant increase in the proportion of NRE::GFP labelled acmcs (38.32\%), up from $17.5 \%$ in $\mathrm{msi}^{1 /+}$ controls (Fig. 4i; Chi-square $\mathrm{p}=.013)$. Strikingly, we observed $52.1 \%$ NRE-GFP positive acmcs and ECs combined in $m \mathrm{si}^{1 / 2}$ transheterozygotes ( $\mathrm{N}=32$ ), up from $22.5 \%$ in $\mathrm{msi}^{1 /+}$ controls ( $\mathrm{N}=22$ ) (Fig. 4i; Chi-square $\mathrm{p}=.0007)$. While we observed a decrease in the percentage of NRE::GFP labelled $m s i^{1 / 2} \mathrm{pcmcs}$ (19.16\%), down from $32.5 \%$ in controls, this result was not statistically significant (Chi-square $p=.067)$. These findings support our lineage tracing and clonal study results, suggesting that loss of Msi function results in preferential differentiation toward an anterior fate.

Due to the altered differentiation potential of FSCs and their daughters within the FSC niche region of msi mutant ovaries, we hypothesised that loss of Msi function may alter somatic cell cycle dynamics within the FSC niche region. To determine this, we used the Drosophila Fluorescence Ubiquitin-based Cell Cycle Indicator (Fly-FUCCI) system, in which degradable versions of GFP::E2f1 and RFP::CycB fluorescently label cells in G1, S and G2 phases of interphase (Zielke et al., 2014). In the fly FUCCI system, G1 is distinguished by GFP::E2F1 labelling in the absence of RFP::CycB. G2 cells appear yellow as they are labelled with both GFP and RFP, and cells in S phase are labelled by RFP alone. The FSC niche driver (10930-Gal4) was used to drive a Gal4 responsive UAS-fly-FUCCI transgene (UAS ${ }^{\mathrm{FUCCI}}$ ) in somatic cells within 
the niche region (Fig. 5a). Counting cells from region 1 to region $2 b$ and excluding mature follicle cells, under heterozygous control conditions $\left(\mathrm{msi}^{2 /+}\right)$, we determined there to be an average of $2.65 \pm .27$ (SEM) G1 labelled cells ( $\mathrm{N}=32$ ) (Fig. $5 \mathrm{~b}$ ) and a total of $94 \%$ of control germaria exhibited at least one cell in G1 phase (Fig. 5b'). A significant reduction in the overall average number of $\mathrm{G} 1$ cells was observed in both $m \mathrm{si}^{2 / 2}(1.85 \pm .23 ; \mathrm{N}=33)$ homozygotes and $m s^{1 / 2}$ transheterozygotes (1.613 $\pm .24 ; \mathrm{N}=31$ ) (Fig. 5b). Despite this result, Chi-square analysis revealed no significant change in the overall proportion of germaria containing at least one cell in G1 phase between control and msi mutant germaria (Fig. 5b'). In contrast, we observed a significant increase in the overall average number of somatic cells in G2 phase in the FSC niche region of both $\mathrm{msi}^{2 / 2}$ and $m \mathrm{si}^{1 / 2}$ mutants (2.85 \pm .27 and $3.23 \pm .25$ respectively) compared to control germaria (.28 \pm .25 ) (Fig. 5c). Strikingly, we counted at least one G2 cell in $91 \%$ of $m s i^{2 / 2}$ germaria and $97 \%$ of $m s i^{1 / 2}$ germaria, while only $19 \%$ of control germaria contained a somatic cell in $\mathrm{G} 2$ phase (Fig. $5 c^{\prime}$ ). Owing to the small sample sizes and the documented short S phase of FSC and their daughters (Skora \& Spradling, 2010), we rarely saw somatic cells in S phase in control germaria (0/32), $m s^{2 / 2}$ mutant germaria (5/33) or $m s^{1 / 2}$ germaria $(2 / 31)$. Overall, these results indicate that absence of Msi function from the FSC niche region results in a $\mathrm{G} 2$ lag in somatic cells in this region.

Studies have shown that FSC proliferation is affected in the absence of sufficient nutrients (Drummond-Barbosa \& Spradling, 2001; Hartman et al., 2010). Because $m s^{2 / 2}$ and $m s i^{1 / 2}$ mutants are less fit than their wild-type counterparts, we wanted to confirm that the observed cell cycle lag in msi mutants was not a result of nutrient deprivation due to poor feeding. We repeated the FUCCl experiment by expressing a $m s^{i N A i}$ transgene in the FSC niche region with 10930-Gal4 concomitantly with the UAS-fly-FUCCI transgene. Consistent with our 

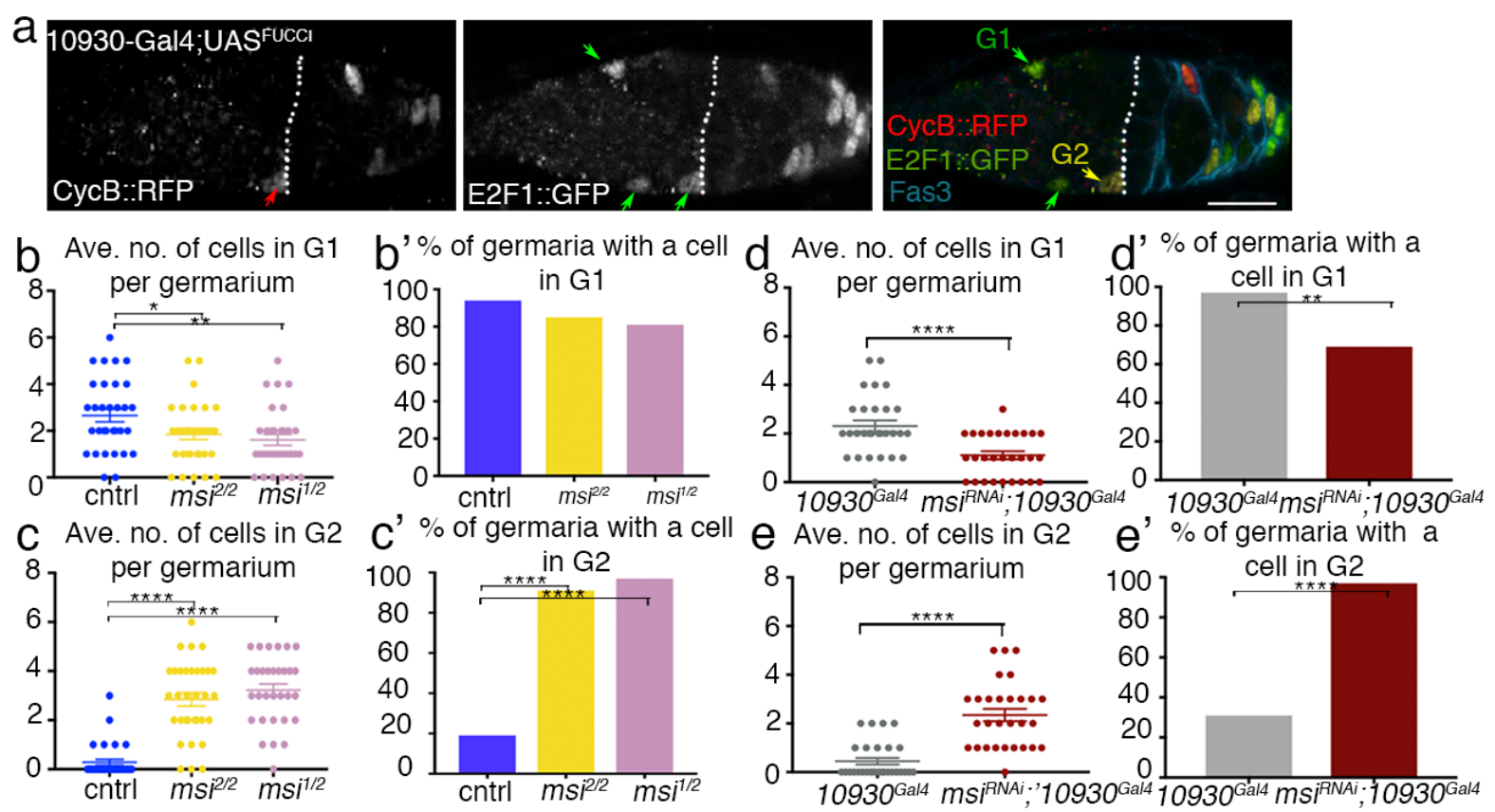

Figure 5. Loss of Msi function from cells within the FSC niche causes cells to lag in G2 phase of the cell cycle. (a) Confocal micrograph showing a representative image of a 10930-Gal4; UAS ${ }^{\mathrm{FUCCl}}$ adult germarium 3 days post eclosion. CycB::RFP-positive cells (red arrow, first panel), E2F1::GFP positive cells (green arrows, second panel), G2 cells (yellow arrow in merge) and $\mathrm{G} 1$ cells (green arrow in merge) all anterior to region 2B boundary (dotted line) are labelled. Scale bar, $10 \mu \mathrm{m}$. (b) Scatterplot of the average number of cells ( \pm SEM) in G1 from region 1 to $2 \mathrm{~b}$ of $\mathrm{msi}^{2 /+}$ (blue), $\mathrm{msi}^{2 / 2}$ (yellow) and $m \mathrm{si}^{1 / 2}$ (pink) germaria showing a significant decrease in the average number of cells in $\mathrm{G} 1$ between control (2.66 $\pm .27 ; \mathrm{N}=32$ ) and both

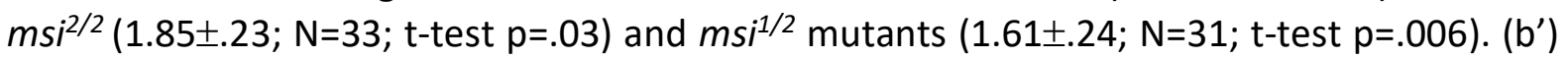
Column graph showing the $\%$ of all control $(94 \%), m s^{2 / 2}(85 \%)$ and $m s^{1 / 2}(81 \%)$ germaria with the presence of at least one cell in G1. (c) Scatterplot depicting the average ( \pm SEM) number of cells in $\mathrm{G} 2$ phase per individual control, $m \mathrm{si}^{2 / 2}$ and $m \mathrm{si}^{1 / 2}$ germarium. A significant increase in the average number of cells in $\mathrm{G} 2$ between control $\left(.28 \pm .25 ; \mathrm{N}=32\right.$ ) and both $\mathrm{msi}^{2 / 2}$ $(2.85 \pm .27 ; \mathrm{N}=33)$ and $m s^{1 / 2}$ mutants $(3.23 \pm .25 ; \mathrm{N}=31$ ) was observed (t-test $\mathrm{p}<.0001$ in both cases). ( $\left.c^{\prime}\right)$ Column graph showing a significant increase in the $\%$ of $m s^{2 / 2}(91 \%$; $N=33$; Chisquare $\mathrm{p}<.0001)$ and $m \mathrm{si}^{1 / 2}(97 \% ; \mathrm{N}=31$; Chi-square $\mathrm{p}<.0001)$ mutant germaria with the presence of at least one cell in $\mathrm{G} 2$ when compared to control germaria. (d) Scatterplot showing a significant difference in the average number of cells ( \pm SEM) in G1 from region 1 $2 \mathrm{~b}$ of $10930^{\mathrm{Gal} / 4}(2.31 \pm 0.23, \mathrm{~N}=29)$ and $\mathrm{msi}^{\text {RNAi; }} 10930^{\mathrm{Gal} / 4}$ germaria $(1.18 \pm 0.16, \mathrm{~N}=29)$ (t-test $\mathrm{p}<.0001)$ and $\left(\mathrm{d}^{\prime}\right)$ column graph showing a significant decrease in the $\%$ of all $\mathrm{msi}^{\text {RNAi}} ; 10930^{\text {Gal }}$ germaria (with the presence of at least one cell in $\mathrm{G1}(69 \%$; $N=29$; Chi-square $\mathrm{p}=.005)$ when compared to $10930^{\text {Gal4 }}$ germaria (97\%). (e) Scatterplot showing a significant difference in the average number of cells $( \pm S E M)$ in $G 2$ phase from region $1-2 b$ of $10930^{\text {Gal4 }}(.45 \pm 0.14, N=29)$ and $\mathrm{msi}^{\text {RNAi}} ; 10930^{\mathrm{Gal} 4}$ germaria $(2.21 \pm 0 . .26, \mathrm{~N}=28)(\mathrm{t}$-test $\mathrm{p}<.0001)$ and $\left(\mathrm{e}^{\prime}\right)$ column graph showing significant increase in the \% of msi $^{\text {RNAi }}$; $10930^{\text {Gal4 }}$ (97\%; $\mathrm{N}=29$; Chi-square $\mathrm{p}<.0001$ ) mutant germaria with the presence of at least one cell in $\mathrm{G} 2$ compared to control germaria (31\%). 
findings using homozygous and transheterozygous msi mutants, knockdown by RNAi resulted in a significant reduction in the average number of cells in $\mathrm{G} 1$ phase per germaria (Fig. $5 \mathrm{~d}$ ). Chi-square analysis in this case also revealed a reduction in the proportion of $m s^{\text {RNAi; }}$,10930Gal4 mutant germaria containing at least one cell in G1 phase ( $p=.005 ;$ Fig. 5d'). Knockdown of Msi by RNAi resulted in a significant increase in the average number of cells in G2 per germaria when comparing to controls (t-test $p<.0001$; Fig. 5e). Impairment of Msi function by RNAi also resulted in a significant increase in the total number of germaria exhibiting at least one somatic cell in $\mathrm{G} 2$ phase (Chi-square $\mathrm{p}<.0001$; Fig. 5e'). Together these results support our findings that loss of Msi function from the ovarian FSC niche results in a G2 lag in somatic cells.

Previous studies have suggested that the combination of graded distributions of JAK-STAT, Wnt and Hh pathway ligands is instrumental in setting the anterior-posterior position of FSCS (Vied et al., 2012). An increase in anterior escort cell differentiation from the FSC niche region has previously been associated with increased Wnt pathway activity (Reilein et al., 2017). Upon identifying a similar phenotype when Msi function is lost from the FSC niche region, we subsequently tested whether genetically modulating Wnt pathway activity could enhance the anterior differentiation phenotype observed in msi mutants. Using 10930-Gal4 ReDDM lineage tracing analysis, we knocked down Msi function in the FSC niche region in a heterozygous $a x n^{E 77}$ mutant background and quantified the number of $\mathrm{H} 2 \mathrm{~B}:$ :RFP expressing cells anterior to region 2a (Fig. 6a-b). Mutations in Axin, a negative regulator of the Wnt signalling pathway, lead to increased Wnt pathway activity (Hamada et al., 1999). Strikingly, the average number of $\mathrm{H} 2 \mathrm{~B}: \mathrm{RFP}$ positive cells anterior to region $2 \mathrm{a}$ increased from $9.12 \pm 1.01 \quad(\mathrm{~N}=25)$ in

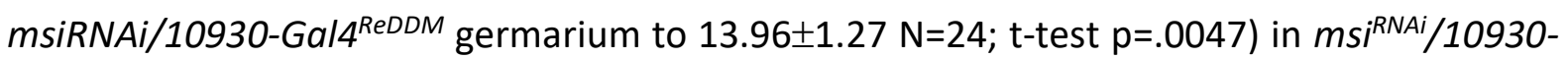


Gal4 ${ }^{R e D D M} ; a x n^{E 77}$ mutant flies (Fig. $6 a$ and $\left.b^{\prime \prime}-b^{\prime \prime \prime}\right)$. In contrast, the average number of H2B::RFP positive cells anterior to region $2 \mathrm{a}$ in control and heterozygous $a x n^{E 77}$ was not significantly different to each other $(5.167 \pm .420$ and $5.783 \pm .489$ respectively) (Fig. 6a and b$\left.b^{\prime}\right)$. These results suggest that somatic cells within the FSC region lacking Msi function are susceptible to even small changes in Wnt signalling output.

We next tested whether loss of Msi function altered Wnt signalling activity. We used the frizzled 3 (fz3)-RFP Wnt activity reporter, which has been noted to be the best available reporter for Wnt signalling activity in the ovary. Reports have shown that Fz3-RFP is expressed highly in regions $1-2 a$, with expression declining in region $2 b$ and rarely detected in follicle cells expressing high levels of Fas3 (Dai et al., 2017; X. Wang \& Page-McCaw, 2014). Fz3-RFP reporter activity in Fas3 positive somatic cells posterior to the FSC niche was observed in $23.8 \%$ of $m s^{1 / 2} 1-2$ day old transheterozygote germarium $(\mathrm{N}=21)$. By comparison, this was observed in only $5.9 \%$ of controls $(\mathrm{N}=17)$ (Fig. $6 \mathrm{c}-\mathrm{d}$ ). These results demonstrate that the output of Wnt signalling activity can be altered upon loss of Msi function in somatic cells within the FSC niche, and consequently support a requirement for Msi in either directly or indirectly modulating Wnt activity in this niche region. Moreover, our results are consistent with previous findings that showed increased Wnt signalling can indeed lead to an increase in escort cell differentiation from the FSC niche (Reilein et al., 2017). 


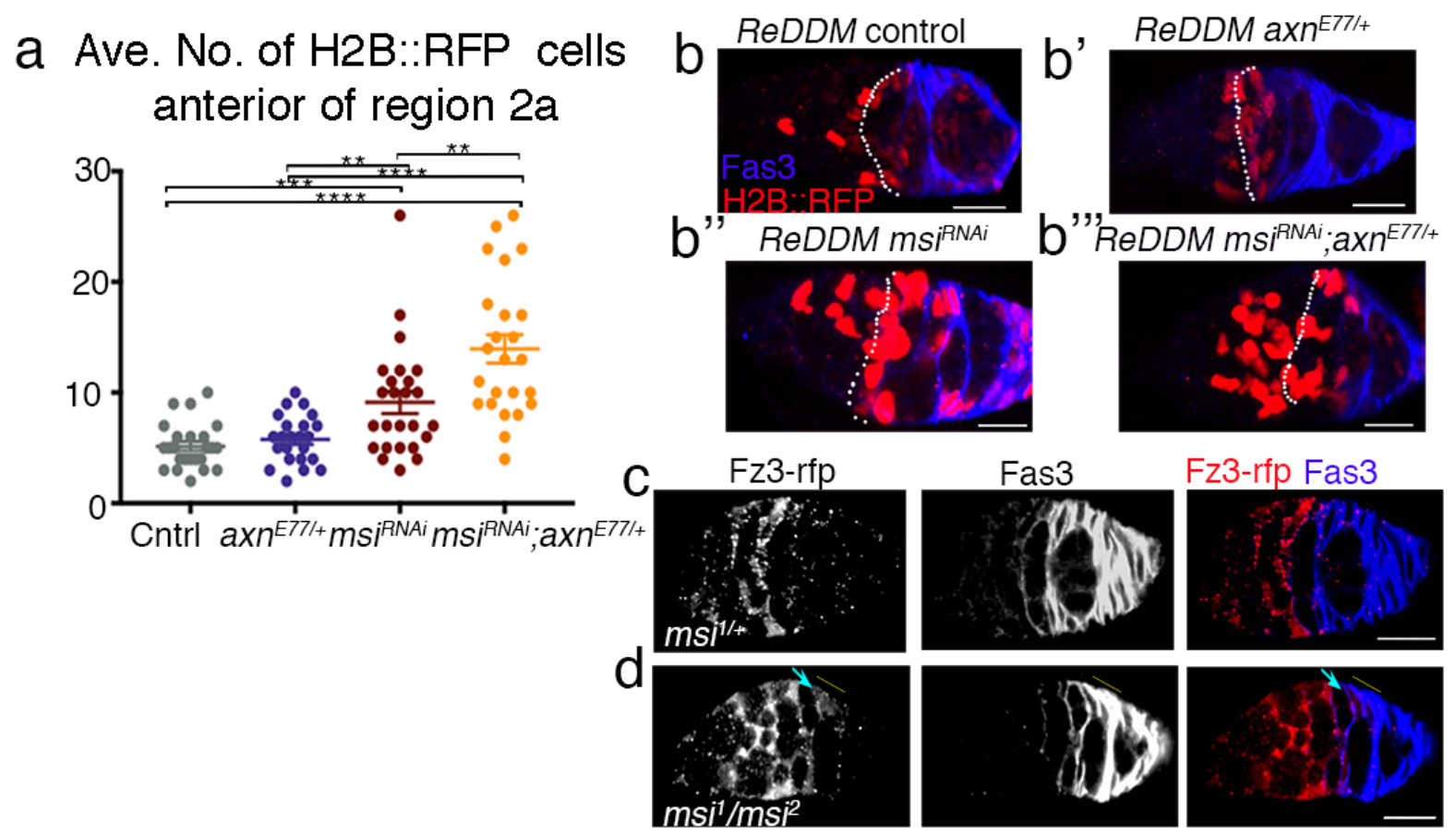

Figure 6. Musashi may maintain FSC niche homeostasis through regulation of Wnt pathway activity. (a) Scatterplot depicting the average number of $\mathrm{H} 2 \mathrm{~B}:: \mathrm{RFP}$ cells ( $\pm \mathrm{SEM}$ ) anterior to region $2 \mathrm{a}$ in germarium from $10930-$ Gal4 ${ }^{\text {REDDM }}(5.167 \pm .420 ; \mathrm{N}=23$; grey), 10930Gal4 $^{\text {REDDM }} ; a_{x n^{E 77 /+}}\left(5.783 \pm .489 ; \mathrm{N}=23\right.$; navy), $\mathrm{msi}^{\text {RNAi }} / 10930-$ Gal $^{\text {REDDM }}(9.12 \pm 1.01 ; \mathrm{N}=24 ;$ red) and $m s i^{R N A i} / 10930-G a l 4^{R E D D M} ; a x n^{E 77 /+}(13.96 \pm 1.27 \mathrm{~N}=21$; orange) flies. t-test significance values are represented by $* * \mathrm{P} \leq 0.01, * * * \mathrm{P} \leq 0.001$ or $* * * * \mathrm{P} \leq 0.0001$. (b-b'") Confocal micrographs of ReDDM lineage tracing in control (b), $a x n^{E 77 /+}\left(b^{\prime}\right), m s i^{R N A i}\left(b^{\prime \prime}\right)$ and $m i^{R N A i} ; a x n^{E 77 /+}\left(b^{\prime \prime \prime}\right)$ germaria. H2B::RFP (red) is projected over multiple $z$ sections, while the Fas 3 channel (blue) is a single slice overlayed image, best representing the $2 b$ boundary (dotted line). (c-d) Fz3-RFP reporter activity in a $\mathrm{msi}^{1 /+}$ control germaria (c) and a $m \mathrm{si}^{1 / 2}$ mutant (d). Blue arrow points to Fz3-RFP expression in mature follicle cells and yellow line indicates continuation of that expression in mature follicle cells expressing Fas3. 


\section{Discussion}

Drosophila Msi was originally identified by its essential role in determining the fate outcome of ectodermal sensory organ precursor cells (Nakamura et al., 1994). Subsequently, Msi has been shown to play a role in photoreceptor cell differentiation (Hirota et al., 1999) and is required for the maintenance of spermatogonial germline stem cells (Siddall et al., 2006). In this study, we have uncovered a role for Msi in regulating the differentiation outcomes of epithelial cells derived from the FSC niche region in the adult ovary. Moreover, our novel findings demonstrate that Msi is required to maintain the fate of epithelial FSCs and/or FPCS and plays an important role in FSC niche homeostasis. Our results, highlighting the concomitant loss of $m s^{i 1}$ mutant FSC clones and gain of EC clones, coupled with ReDDM lineage tracing experiments, indicate that loss of Msi from the niche results in a shift in the differentiation of FSCs/FPCs toward an anterior EC fate. These results are consistent with a previous study that demonstrated through live cell imaging and clonal analysis that somatic cells from within the FSC region can produce more anterior ECs (Reilein et al., 2017).

A distortion in the lateral cross-migration of FSC progeny may provide some clarification into how an increase in the production of anterior ECs from the FSC niche can occur under loss of Msi function conditions. FSC daughters reportedly alternate between the generation of a cross-migrating cell $(\mathrm{cmc})$, that migrates along the anterior aspect of the first region $2 \mathrm{~b}$ cyst, and a posterior-migrating cell (pmc), the progeny of which migrate across the posterior aspect of the same region $2 b$ cyst (Nystul \& Spradling, 2007). Clonal experiments have revealed this anterior or posterior positional bias occur at equivalent frequencies, and research has shown the ability of FSC daughters to migrate laterally is dependent upon Notch signalling (Nystul \& Spradling, 2010). In msi mutants, we observed a significant increase in cells expressing NRE- 
GFP positioned anteriorly along the region $2 \mathrm{~b}$ face in the germarium. Although not statistically significant, we also observed a trend against msi mutant pcmcs. This may represent an increased anterior bias of FSC daughters, positioning them toward differentiation to an anterior fate.

The complex intersection of signalling pathways is involved in FSC differentiation and specification, and the increase in the number of anteriorly positioned msi mutant FSCs/FPCS within the germarium may predispose cells to receiving signals which direct cells toward an anterior fate. Although Notch, Hh, Wnt, Hippo and EGFR signalling all play roles in some aspect of FSC proliferation, FSC self-renewal or differentiation, FSC survival and competition (reviewed in (Rust \& Nystul, 2020)), we specifically addressed whether msi mutations may affect Wnt signalling activity, since an increase in Wnt activity has recently been associated with an increase in the differentiation towards an anterior EC fate from the FSC niche (Reilein et al., 2017). A steep gradient of Wnt signalling activity has been reported in the germarium from regions 1 to $2 b$, with high levels of Wnt signalling activity present in FSCs and FPCs at the region $2 a / 2 b$ border, while nearly undetectable levels of Wnt pathway activity have been reported in FPCs that have moved into Region 2b of the germarium (Dai et al., 2017; Kim-Yip \& Nystul, 2018). When we introduced a heterozygous loss of function axin allele, a negative regulator of the Wnt pathway, into a genotype where Msi function was reduced in the FSC niche region by way of RNAi, lineage tracing experiments showed a genetic enhancement of the msi mutant phenotype, with a further increase in $\mathrm{H} 2 \mathrm{~B}$-expressing somatic cells having arisen from the niche. These results may indicate that anterior-biased msi mutant FPCs are more susceptible to changes in the levels of Wnt signalling. This hypothesis is supported by a previous study which suggested that FSCs/FPCs that sit anteriorly within the niche are 
directed toward an anterior fate upon elevated levels of Wnt signalling activity (Reilein et al., 2017). In our study, we also documented the mis-expression of the FZ3-RFP reporter in msi mutant cells posterior to region $2 b$, suggesting that loss of Msi function directly or indirectly alters Wnt signalling activity, which may also, in turn, impact the fate outcome of somatic cells within the niche region. It is important to note that Msi, being an RNA-binding protein, is likely to regulate multiple targets. While not the aim of this study, it is highly likely that multiple signalling pathways have been altered through abrogation of Msi function. The combinatorial effect of changes to multiple signalling components in a context-dependent manner in msi mutants may determine differentiation outcomes of cells and further studies are required to confirm this hypothesis.

Loss of Msi function from the somatic cells within the FSC niche resulted in a change in cell cycle dynamics, with an increased proportion of mutant somatic cells in this region found to be lagging in G2 phase of the cell cycle. More research is required to determine whether this observed G2 lag in msi mutants predisposes stem-like cells within the FSC niche to preferentially differentiate and acquire a specific fate. Epithelial stem cell differentiation of G2 arrested cells has been reported in the freshwater vertebrate Hydra, a non-human cancer model system, where a proportion of epithelial stem cells terminally differentiate in G2 phase of the cell cycle (Buzgariu, Crescenzi, \& Galliot, 2014). In Drosophila sensory development, primary progenitor cells arrest in G2 phase, the purpose of which is thought to prevent progenitor self-renewal and allow synchronization of cell division with cell fate differentiation (Ayeni et al., 2016). A recent study has shown that quiescent Drosophila renal stem cells are arrested in G0 or G2 (C. Wang \& Spradling, 2020). Interestingly, the authors proposed that G2-arrested quiescent renal stem cells would be well positioned to respond promptly to 
injury, highlighting the capacity of cells in $G 2$ phase to respond to developmental cues or signalling pathway changes (C. Wang \& Spradling, 2020). Significantly, research has shown that $\mathrm{Wnt} / \beta$-catenin signalling is under cell cycle control and peaks at $\mathrm{G} 2 / \mathrm{M}$ phase (Davidson et al., 2009; Ding et al., 2014). While the reason for the observed G2 lag in msi mutant FSC/FPCs remains unknown, an increase in Wnt activity and subsequent skew towards EC differentiation could be an indirect consequence.

One further aspect to come out of our research is the notion that some ECs differentiate from FSCs/FPCs within the niche region under normal conditions. This has also been observed in a previous study (Reilein et al., 2017). The demarcation between posterior ECs and FPCs/FSCs remains difficult to determine. Recent analyses of ECs have revealed distinct differences of EC populations depending on their position within the germarium. Anterior ECs are morphologically distinct from more posterior ECs, displaying short and less elaborate extensions, while posterior ECs typically show longer and more stable projections (Banisch et al., 2017). Additional evidence shows that posterior ECs divide in a Wnt6-dependent manner to replace anterior cells that have died, while more anterior ECs remain quiescent in G1 (X. Wang \& Page-McCaw, 2018). These reports suggest that posterior ECs are more stem-like in nature, having the capacity to divide and differentiate, with proper differentiation being highly dependent upon the signals they receive from surrounding cells. While our results demonstrate that FSCS/FPCs prematurely differentiate toward an anterior fate in msi mutants, we cannot completely rule out the possibility of an increase in posterior EC migration to more anterior positions within the germarium, an event independent of the FSC loss that we observed. 
Our finding that Msi is required to maintain the epithelial stem/precursor fate is consistent with the known function of its vertebrate orthologues, Msi-1 and Msi-2. Vertebrate Msi-2 has been shown to play a critical role in hematopoietic cell fate and lineage bias (Park et al., 2014). Vertebrate Msi proteins have been shown to be associated with epithelial cell state in several cancer types, most notably breast cancer, with loss of Msi leading to loss of epithelial cell identity (Katz et al., 2014). In the intestinal epithelium, a requirement for vertebrate Msi proteins in maintaining quiescent intestinal stem cells has been demonstrated (Yousefi et al., 2016). Interestingly, vertebrate Musashi proteins have been shown to interact with the Notch, Transforming Growth Factor Beta (TGF-ß), Wnt and Hh signalling pathways in differing contexts (reviewed in (das Chagas, Baroni, Brassesco, \& Tone, 2020)). Here we describe a specific context where Drosophila Msi is required for epithelial stem cell homeostasis and demonstrate an interaction between Msi and the Wnt signalling pathway. Importantly, this research provides a model in which to further our understanding of tissue repair in disease and post cytotoxic treatment, and investigate the molecular mechanisms underpinning the maintenance of epithelial stem cells in a context dependent manner. 


\section{Materials and Methods}

\section{Fly Strains}

All flies were raised on standard molasses-based food at $25^{\circ} \mathrm{C}$ except for Gal4 crosses, which

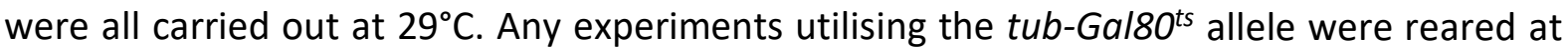
$18^{\circ} \mathrm{C}$ before being shifted to $29^{\circ} \mathrm{C}$ upon eclosion. Fly stocks used in this study include $w^{1118}$, $m s^{1}, m s i^{2}, F r t 82 B m s i^{1}, F r t 82 B m s i^{2}, m s i^{R N A i}\left(2^{\text {nd }}\right.$ chromosome insert, Bloomington stock \#55152), 10930-Gal4, UAS-mcD8::GFP (CD8::GFP) reporter on $1^{\text {st }}$ and $3^{\text {rd }}$ chromosomes, NREGFP (NRE::GFP) on $3^{\text {rd }}$ chromosome, UAS-FUCCI transgenes (Bloomington stocks \#55110 \#55111), FRT82BUbi-GFP and Frt42D;FRT82B, all of which are available from the Bloomington Stock Center, Indiana. The $\mathrm{X}$ chromosome $m s^{i R N A i}$ fly strain was obtained from the Vienna Drosophila Resource Center (\#11784). Other fly strains used include fz3-RFP reporter (gift from Page-McCaw lab, Vanderbilt School of Medicine, Nashville), fax-GFP reporter (gift from Xie lab, Stowers Institute for Medical Research, Missouri, USA), frt82B; $a x n^{E 77}$ (gift from Richardson lab, La Trobe University, Bundoora Australia) and ReDDM strain tub-Gal80 Histone2B::RFP (gift from Murray lab, University of Melbourne).

\section{Immunostaining and image analysis}

Appropriately aged females were dissected in PBS, fixed for 20 minutes in 4\% Formaldehyde diluted in PBT (PBS +.2\% Triton X-100 (Sigma)), washed for $3 \times 10$ minutes in PBT and then incubated for 45 minutes in PBTH (5\% Normal Horse Serum diluted with PBT). Ovaries were then incubated overnight at $4^{\circ} \mathrm{C}$ in primary antibodies diluted in PBT. Samples were washed a further 3x10 minutes in PBS before secondary antibody incubation was carried out for 2 hours at room temperature in PBT. Samples were washed for a further $3 \times 10$ minutes before ovaries were mounted on slides in Prolong ${ }^{\mathrm{TM}}$ Gold Antifade Reagent with Dapi (Invitrogen). 
Antibodies used in this study include 1:10 rat anti-Msi (gift of $\mathrm{H}$. Okano, Keio University), 1:20 mouse anti-FasIII (Developmental Studies Hybridoma Bank (DSHB)), 1:100 goat anti-Vasa (Santa Cruz Biotechnology), 1:2000 chicken anti-GFP (Abcam), 1:500 rabbit anti-RFP (Invitrogen), 1:10 mouse anti 1B1 (DSHB), 1:20 mouse anti-Bam (DSHB), 1:100 mouse antichickadee (DSHB), 1:10 guinea pig anti-Traffic Jam (gift of Dorothea Godt, University of Toronto). Secondary antibodies (Donkey anti-Mouse Alexa Fluor 488/ 594/ 647, Donkey antiRabbit Alexa Fluor 594, Donkey anti-Rat Alexa Fluor 488/594) were obtained from (Thermo Fisher Scientific) and used at a dilution of 1:500. Donkey anti-guinea pig 594, and donkey-anti chicken 488 were obtained from Jackson Immuno Research Labs and used at a dilution of 1:500. Images were acquired on Zeiss LSM800 or LSM880 confocal microscopes as serial optical sections (z-stacks) optimized to acquire overlapping sections. FIJI/ ImageJ was then used to process images, add scale bars and analyse pixel intensity for REDDM analysis. Orthogonal projections in Figure 2 were produced in FIJI. Control and Msi mutant Fz3-RFP expression in Figure 6 were adjusted simultaneously to reduce background noise in Adobe Photoshop. Photoshop was used to compile figure panels.

\section{Mosaic analysis}

Negatively marked GSC and FSC clones were induced by Flp-FRT in 2-3 day old females of genotypes $h s$-Flp/+; FRT82B msi ${ }^{1}$ /FRT82B Ubi-GFP, $h s$-Flp/+; FRT82B $m s^{2} /$ FRT82B Ubi-GFP, hs-Flp/+; FRT42D/+;FRT82B/FRT82B Ubi-GFP. Well-fed females were heat shocked in a water bath at $37^{\circ} \mathrm{C}$ for 1 hour, twice a day for 2 consecutive days, with a recovery period of $8 \mathrm{hrs}$ between heat shocks. Ovaries dissected from 7 and 21 day old females (post heat shock) were stained with anti-GFP, anti-Traffic Jam and anti-Fas3. GFP-negative but TJ positive clones at 
the $2 a / 2 b$ boundary were counted as FSC clones. GFP-negative but TJ positive clones in the region anterior to the $2 \mathrm{a} / 2 \mathrm{~b}$ boundary were counted as $\mathrm{EC}$ clones.

\section{ReDDM lineage tracing analysis}

Flies were raised at $18^{\circ} \mathrm{C}$ and shifted to $29^{\circ} \mathrm{C}$ for 14 days before dissection. Serial overlapping optical sections were analysed with FIJI, where the ROI manager was used to select $\mathrm{H} 2 \mathrm{~B}:$ :RFP positive cells and only cells with an average brightness threshold of 120 or more were counted. Optical sections were carefully analysed in conjunction with Dapi and with the ROI manager to ensure that cells were not counted more than once. Full genotypes of flies used in ReDDM analysis include +/+; 10930-Gal4/CyO; tub-Gal80 ${ }^{\text {ts }}$, UAS-Histone2B::RFP/UASCD8::GFP (10930-Gal4 ReDDM, Fig. 4 panel a \& c), +/+; 10930-Gal4/msi ${ }^{\text {RNAi }}$; tub-Gal80ts, UASHistone2B::RFP/UAS-CD8::GFP (10930-Gal4 ReDDM/msi ${ }^{R N A i}$, Fig. 4 panel b \& c), UAS-CD8::GFP/+; 10930-Gal4/+; tub-Gal80ts , UAS-Histone2B::RFP /+ (ReDDM control Fig. 6 panel a \& b), UASCD8::GFP/+; 10930-Gal4/+; tub-Gal80's, UAS-Histone2B::RFP/axn ${ }^{E 77}$ (ReDDM axn ${ }^{E 77 /+}$ panel a and b') UAS-CD8::GFP/+; 10930-Gal4/msi ${ }^{\text {RNAi; }}$ tub-Gal80's, UAS-Histone2B::RFP /+ (ReDDM $m s^{R N A}$, Fig. 6 panel a \& $\left.b^{\prime \prime}\right)$, UAS-CD8::GFP/+; 10930-Gal4/msi ${ }^{\text {RNAi; }}$ tub-Gal80 ${ }^{\text {ts }}$, UASHistone2B::RFP / $a x n^{E 77}$ (ReDDM msi ${ }^{R N A i} ; a x n^{E 77 /+}$ Fig. 6 panel a \& $\left.b^{\prime \prime \prime}\right)$.

\section{NRE-GFP analysis}

$m s^{1}$ was recombined onto the same chromosome as the NRE::GFP transgene. Serial overlapping optical sections were analysed in FIJI to obtain the total number of GFP-positive cells from region 1 to 3 of the germaria as well as for the anatomical position of each GFP positive cell. Full genotypes of flies used in analysis include 10930-Gal4/UAS-RFP; 
NRE::GFP, $m s i^{1} /+$ (control, Fig. 4 panels $d, e, f, h$ and i) and 10930-Gal4/UAS-RFP; NRE::GFP, $m s i^{1} / \mathrm{msi}^{2}\left(m s i^{1} / m s i^{2}\right.$, Fig. 4 panels $\mathrm{g}$, $\mathrm{h}$ and i). $m \mathrm{si}^{1}$ was recombined onto the same chromosome as the NRE::GFP transgene.

\section{FUCCI analysis}

Flies were raised at $25^{\circ} \mathrm{C}$ and shifted to $29^{\circ} \mathrm{C}$ upon eclosion for 3 days. Well-fed females were dissected and stained for detection of GFP and RFP. Serial overlapping optical sections were analysed in FIJI, with Dapi used in conjunction with the ROI manager to make sure to not duplicate cell counts. The minimal brightness threshold utilised for the RFP and GFP channels was 110. Full genotypes of flies used in $\mathrm{FUCCl}$ analysis include 10930-Gal4/UASp-GFP::E2f, UASp-mRFP1.NLS::CycB; $m s^{2}{ }^{2} /+$ (panel a and cntrl in Fig. 5b-c'), 10930-Gal4/UASp-GFP::E2f, UASp-mRFP1.NLS::CycB; $m s i^{2 / 2}\left(m s i^{2 / 2}\right.$ in Fig. 5b-c'), 10930Gal4/UASp-GFP::E2f, UASp-mRFP1.NLS::CycB; $m s^{1} / m s i^{2}\left(m s i^{1} / m s i^{2}\right.$ in Fig. 5b-c' $),+/+; 10930-$ Gal4/+; UASp-GFP::E2f, UASp-mRFP1.NLS::CycB/+ (10930 Gal4 in Fig. 5d-e), msi ${ }^{\text {RNAi } /+; ~ 10930-~}$ Gal4/+; UASp-GFP::E2f, UASp-mRFP1.NLS::CycB (msi ${ }^{\text {RNAi; } 10930 G a l 4}$ in panels Fig. 5d-e').

\section{Statistics}

Statistical analyses were performed using Graphpad Prism. P-values have been acquired through Chi-square analysis or obtained by Welch's t-test, as stipulated in figure legends and text. All scatterplots are graphed showing the mean \pm SEM. 


\section{Acknowledgements}

The authors wish to thank Andrea Page-McCaw, Ting Xie, Helena Richardson, Michael Murray, Leonie Quinn, Bloomington Drosophila Stock Center, Vienna Drosophila RNAi Center and the Australian Drosophila Biomedical Research Support Facility (OzDros) for provision of Drosophila strains. We also wish to thank Hideyuki Okano, Dorothea Godt and the Developmental Studies Hybridoma Bank for provision of antibodies. We are grateful for the assistance of the Biomedical Optical Microscopy Platform at the University of Melbourne. This work was conducted with the support of Australian Research Council Discovery Project Grants 12010024 and 170102379 to $\mathrm{GH}$.

\section{Competing Interests}

The authors have no competing interests to declare. 


\section{References}

Amoyel, M., Simons, B. D., \& Bach, E. A. (2014). Neutral competition of stem cells is skewed by proliferative changes downstream of Hh and Hpo. EMBO J, 33(20), 2295-2313. doi:10.15252/embj.201387500

Antonello, Z. A., Reiff, T., Ballesta-Illan, E., \& Dominguez, M. (2015). Robust intestinal homeostasis relies on cellular plasticity in enteroblasts mediated by miR-8-Escargot switch. EMBO J, 34(15), 2025-2041. doi:10.15252/embj.201591517

Asai, R., Okano, H., \& Yasugi, S. (2005). Correlation between Musashi-1 and c-hairy-1 expression and cell proliferation activity in the developing intestine and stomach of both chicken and mouse. Dev Growth Differ, 47(8), 501-510. doi:10.1111/j.1440169X.2005.00825.X

Ayeni, J. O., Audibert, A., Fichelson, P., Srayko, M., Gho, M., \& Campbell, S. D. (2016). G2 phase arrest prevents bristle progenitor self-renewal and synchronizes cell division with cell fate differentiation. Development, 143(7), 1160-1169. doi:10.1242/dev.134270

Banisch, T. U., Maimon, I., Dadosh, T., \& Gilboa, L. (2017). Escort cells generate a dynamic compartment for germline stem cell differentiation via combined Stat and Erk signalling. Development, 144(11), 1937-1947. doi:10.1242/dev.143727

Buzgariu, W., Crescenzi, M., \& Galliot, B. (2014). Robust G2 pausing of adult stem cells in Hydra. Differentiation, 87(1-2), 83-99. doi:10.1016/j.diff.2014.03.001

Dai, W., Peterson, A., Kenney, T., Burrous, H., \& Montell, D. J. (2017). Quantitative microscopy of the Drosophila ovary shows multiple niche signals specify progenitor cell fate. Nat Commun, 8(1), 1244. doi:10.1038/s41467-017-01322-9

das Chagas, P. F., Baroni, M., Brassesco, M. S., \& Tone, L. G. (2020). Interplay between the RNA binding-protein Musashi and developmental signaling pathways. J Gene Med, 22(1), e3136. doi:10.1002/jgm.3136

Davidson, G., Shen, J., Huang, Y. L., Su, Y., Karaulanov, E., Bartscherer, K., . . Niehrs, C. (2009). Cell cycle control of wnt receptor activation. Dev Cell, 17(6), 788-799. doi:10.1016/j.devcel.2009.11.006

de Navascues, J., Perdigoto, C. N., Bian, Y., Schneider, M. H., Bardin, A. J., Martinez-Arias, A., \& Simons, B. D. (2012). Drosophila midgut homeostasis involves neutral competition between symmetrically dividing intestinal stem cells. EMBO J, 31(11), 2473-2485. doi:10.1038/emboj.2012.106

Ding, Y., Su, S., Tang, W., Zhang, X., Chen, S., Zhu, G., . . . Wu, W. (2014). Enrichment of the beta-catenin-TCF complex at the $\mathrm{S}$ and G2 phases ensures cell survival and cell cycle progression. J Cell Sci, 127(Pt 22), 4833-4845. doi:10.1242/jcs.146977

Drummond-Barbosa, D., \& Spradling, A. C. (2001). Stem cells and their progeny respond to nutritional changes during Drosophila oogenesis. Dev Biol, 231(1), 265-278. doi:10.1006/dbio.2000.0135

Fadiga, J., \& Nystul, T. G. (2019). The follicle epithelium in the Drosophila ovary is maintained by a small number of stem cells. Elife, 8. doi:10.7554/eLife.49050

Hamada, F., Tomoyasu, Y., Takatsu, Y., Nakamura, M., Nagai, S., Suzuki, A., . . . Akiyama, T. (1999). Negative regulation of Wingless signaling by D-axin, a Drosophila homolog of axin. Science, 283(5408), 1739-1742. doi:10.1126/science.283.5408.1739

Hartman, T. R., Ventresca, E. M., Hopkins, A., Zinshteyn, D., Singh, T., O'Brien, J. A., . . . O'Reilly, A. M. (2015). Novel tools for genetic manipulation of follicle stem cells in the Drosophila ovary reveal an integrin-dependent transition from quiescence to proliferation. Genetics, 199(4), 935-957. doi:10.1534/genetics.114.173617 
Hartman, T. R., Zinshteyn, D., Schofield, H. K., Nicolas, E., Okada, A., \& O'Reilly, A. M. (2010). Drosophila Boi limits Hedgehog levels to suppress follicle stem cell proliferation. J Cell Biol, 191(5), 943-952. doi:10.1083/jcb.201007142

Hirota, Y., Okabe, M., Imai, T., Kurusu, M., Yamamoto, A., Miyao, S., . . Okano, H. (1999). Musashi and seven in absentia downregulate Tramtrack through distinct mechanisms in Drosophila eye development. Mech Dev, 87(1-2), 93-101. doi:10.1016/s09254773(99)00143-4

Housden, B. E., Millen, K., \& Bray, S. J. (2012). Drosophila Reporter Vectors Compatible with PhiC31 Integrase Transgenesis Techniques and Their Use to Generate New Notch Reporter Fly Lines. G3 (Bethesda), 2(1), 79-82. doi:10.1534/g3.111.001321

Huang, P., Sahai-Hernandez, P., Bohm, R. A., Welch, W. P., Zhang, B., \& Nystul, T. (2014). Enhancer-trap flippase lines for clonal analysis in the Drosophila ovary. G3 (Bethesda), 4(9), 1693-1699. doi:10.1534/g3.114.010710

Ito, T., Kwon, H. Y., Zimdahl, B., Congdon, K. L., Blum, J., Lento, W. E., . . Reya, T. (2010). Regulation of myeloid leukaemia by the cell-fate determinant Musashi. Nature, 466(7307), 765-768. doi:10.1038/nature09171

Johnston, M. J., Bar-Cohen, S., Paroush, Z., \& Nystul, T. G. (2016). Phosphorylated Groucho delays differentiation in the follicle stem cell lineage by providing a molecular memory of EGFR signaling in the niche. Development, 143(24), 4631-4642. doi:10.1242/dev.143263

Kaneko, Y., Sakakibara, S., Imai, T., Suzuki, A., Nakamura, Y., Sawamoto, K., . . O Okano, H. (2000). Musashi1: an evolutionally conserved marker for CNS progenitor cells including neural stem cells. Dev Neurosci, 22(1-2), 139-153. doi:10.1159/000017435

Katz, Y., Li, F., Lambert, N. J., Sokol, E. S., Tam, W. L., Cheng, A. W., . . . Burge, C. B. (2014). Musashi proteins are post-transcriptional regulators of the epithelial-luminal cell state. Elife, 3, e03915. doi:10.7554/eLife.03915

Kharas, M. G., Lengner, C. J., Al-Shahrour, F., Bullinger, L., Ball, B., Zaidi, S., . . Daley, G. Q. (2010). Musashi-2 regulates normal hematopoiesis and promotes aggressive myeloid leukemia. Nat Med, 16(8), 903-908. doi:10.1038/nm.2187

Kim-Yip, R. P., \& Nystul, T. G. (2018). Wingless promotes EGFR signaling in follicle stem cells to maintain self-renewal. Development, 145(23). doi:10.1242/dev.168716

Kirilly, D., Spana, E. P., Perrimon, N., Padgett, R. W., \& Xie, T. (2005). BMP signaling is required for controlling somatic stem cell self-renewal in the Drosophila ovary. Dev Cell, 9(5), 651-662. doi:10.1016/j.devcel.2005.09.013

Kirilly, D., Wang, S., \& Xie, T. (2011). Self-maintained escort cells form a germline stem cell differentiation niche. Development, 138(23), 5087-5097. doi:10.1242/dev.067850

Klein, A. M., \& Simons, B. D. (2011). Universal patterns of stem cell fate in cycling adult tissues. Development, 138(15), 3103-3111. doi:10.1242/dev.060103

Kronen, M. R., Schoenfelder, K. P., Klein, A. M., \& Nystul, T. G. (2014). Basolateral junction proteins regulate competition for the follicle stem cell niche in the Drosophila ovary. PLoS One, 9(7), e101085. doi:10.1371/journal.pone.0101085

Lopez-Schier, H., \& St Johnston, D. (2001). Delta signaling from the germ line controls the proliferation and differentiation of the somatic follicle cells during Drosophila oogenesis. Genes Dev, 15(11), 1393-1405. doi:10.1101/gad.200901

Maimon, I., Popliker, M., \& Gilboa, L. (2014). Without children is required for Stat-mediated zfh1 transcription and for germline stem cell differentiation. Development, 141(13), 2602-2610. doi:10.1242/dev.109611

Margolis, J., \& Spradling, A. (1995). Identification and behavior of epithelial stem cells in the Drosophila ovary. Development, 121(11), 3797-3807. 
Nakamura, M., Okano, H., Blendy, J. A., \& Montell, C. (1994). Musashi, a neural RNAbinding protein required for Drosophila adult external sensory organ development. Neuron, 13(1), 67-81. doi:10.1016/0896-6273(94)90460-x

Nystul, T., \& Spradling, A. (2007). An epithelial niche in the Drosophila ovary undergoes longrange stem cell replacement. Cell Stem Cell, 1(3), 277-285. doi:10.1016/j.stem.2007.07.009

Nystul, T., \& Spradling, A. (2010). Regulation of epithelial stem cell replacement and follicle formation in the Drosophila ovary. Genetics, 184(2), 503-515. doi:10.1534/genetics.109.109538

O'Reilly, A. M., Lee, H. H., \& Simon, M. A. (2008). Integrins control the positioning and proliferation of follicle stem cells in the Drosophila ovary. J Cell Biol, 182(4), 801-815. doi:10.1083/jcb.200710141

Ohlstein, B., \& McKearin, D. (1997). Ectopic expression of the Drosophila Bam protein eliminates oogenic germline stem cells. Development, 124(18), 3651-3662.

Park, S. M., Deering, R. P., Lu, Y., Tivnan, P., Lianoglou, S., Al-Shahrour, F., . . Kharas, M. G. (2014). Musashi-2 controls cell fate, lineage bias, and TGF-beta signaling in HSCs. J Exp Med, 211(1), 71-87. doi:10.1084/jem.20130736

Potten, C. S., Booth, C., Tudor, G. L., Booth, D., Brady, G., Hurley, P., . . Okano, H. (2003). Identification of a putative intestinal stem cell and early lineage marker; musashi-1. Differentiation, 71(1), 28-41. doi:10.1046/j.1432-0436.2003.700603.x

Reilein, A., Melamed, D., Park, K. S., Berg, A., Cimetta, E., Tandon, N., . . . Kalderon, D. (2017). Alternative direct stem cell derivatives defined by stem cell location and graded Wnt signalling. Nat Cell Biol, 19(5), 433-444. doi:10.1038/ncb3505

Rust, K., \& Nystul, T. (2020). Signal transduction in the early Drosophila follicle stem cell lineage. Curr Opin Insect Sci, 37, 39-48. doi:10.1016/j.cois.2019.11.005

Sahai-Hernandez, P., \& Nystul, T. G. (2013). A dynamic population of stromal cells contributes to the follicle stem cell niche in the Drosophila ovary. Development, 140(22), 4490-4498. doi:10.1242/dev.098558

Sakakibara, S., Nakamura, Y., Yoshida, T., Shibata, S., Koike, M., Takano, H., . . Okano, H. (2002). RNA-binding protein Musashi family: roles for CNS stem cells and a subpopulation of ependymal cells revealed by targeted disruption and antisense ablation. Proc Natl Acad Sci U S A, 99(23), 15194-15199. doi:10.1073/pnas.232087499

Siddall, N. A., McLaughlin, E. A., Marriner, N. L., \& Hime, G. R. (2006). The RNA-binding protein Musashi is required intrinsically to maintain stem cell identity. Proc Natl Acad Sci U S A, 103(22), 8402-8407. doi:10.1073/pnas.0600906103

Simons, B. D., \& Clevers, H. (2011). Strategies for homeostatic stem cell self-renewal in adult tissues. Cell, 145(6), 851-862. doi:10.1016/j.cell.2011.05.033

Skora, A. D., \& Spradling, A. C. (2010). Epigenetic stability increases extensively during Drosophila follicle stem cell differentiation. Proc Natl Acad Sci U S A, 107(16), 73897394. doi:10.1073/pnas.1003180107

Song, X., \& Xie, T. (2003). Wingless signaling regulates the maintenance of ovarian somatic stem cells in Drosophila. Development, 130(14), 3259-3268. doi:10.1242/dev.00524

Spradling, A. C. (1993). Developmental genetics of oogenesis. In M. Bate \& A. Martinez-Arias (Eds.), The Development of Drosophila melanogaster (Vol. 1, pp. 1-70). Cold Spring Harbor, NY: Cold Spring Harbor Laboratory Press.

Stine, R. R., \& Matunis, E. L. (2013). Stem cell competition: finding balance in the niche. Trends Cell Biol, 23(8), 357-364. doi:10.1016/j.tcb.2013.03.001

Sutherland, J. M., Fraser, B. A., Sobinoff, A. P., Pye, V. J., Davidson, T. L., Siddall, N. A., . . . McLaughlin, E. A. (2014). Developmental expression of Musashi-1 and Musashi-2 
RNA-binding proteins during spermatogenesis: analysis of the deleterious effects of dysregulated expression. Biol Reprod, 90(5), 92. doi:10.1095/biolreprod.113.115261

Sutherland, J. M., Sobinoff, A. P., Fraser, B. A., Redgrove, K. A., Davidson, T. L., Siddall, N. A., . . . McLaughlin, E. A. (2015). RNA binding protein Musashi-1 directly targets Msi2 and Erh during early testis germ cell development and interacts with IPO5 upon translocation to the nucleus. FASEB J, 29(7), 2759-2768. doi:10.1096/fj.14-265868

Sutherland, J. M., Sobinoff, A. P., Gunter, K. M., Fraser, B. A., Pye, V., Bernstein, I. R., .. . McLaughlin, E. A. (2015). Knockout of RNA Binding Protein MSI2 Impairs Follicle Development in the Mouse Ovary: Characterization of MSI1 and MSI2 during Folliculogenesis. Biomolecules, 5(3), 1228-1244. doi:10.3390/biom5031228

Vied, C., Reilein, A., Field, N. S., \& Kalderon, D. (2012). Regulation of stem cells by intersecting gradients of long-range niche signals. Dev Cell, 23(4), 836-848. doi:10.1016/j.devcel.2012.09.010

Waghmare, I., \& Page-McCaw, A. (2018). Wnt Signaling in Stem Cell Maintenance and Differentiation in the Drosophila Germarium. Genes (Basel), 9(3). doi:10.3390/genes9030127

Wang, C., \& Spradling, A. C. (2020). An abundant quiescent stem cell population in Drosophila Malpighian tubules protects principal cells from kidney stones. Elife, 9. doi:10.7554/eLife.54096

Wang, X., \& Page-McCaw, A. (2014). A matrix metalloproteinase mediates long-distance attenuation of stem cell proliferation. $J$ Cell Biol, 206(7), 923-936. doi:10.1083/jcb.201403084

Wang, X., \& Page-McCaw, A. (2018). Wnt6 maintains anterior escort cells as an integral component of the germline stem cell niche. Development, 145(3). doi:10.1242/dev.158527

Wang, Y., Jiang, C. Q., \& Fan, L. F. (2015). Correlation of Musashi-1, Lgr5, and pEGFR expressions in human small intestinal adenocarcinomas. Tumour Biol, 36(8), 60756082. doi:10.1007/s13277-015-3288-3

Watt, F. M., \& Hogan, B. L. (2000). Out of Eden: stem cells and their niches. Science, 287(5457), 1427-1430. doi:10.1126/science.287.5457.1427

Yang, Z., Sun, J., Hu, Y., Wang, F., Wang, X., Qiao, H. H., . . Ni, J. Q. (2017). Histone H1 defect in escort cells triggers germline tumor in Drosophila ovary. Dev Biol, 424(1), 40-49. doi:10.1016/j.ydbio.2017.02.012

Yousefi, M., Li, N., Nakauka-Ddamba, A., Wang, S., Davidow, K., Schoenberger, J., . . . Lengner, C. J. (2016). Msi RNA-binding proteins control reserve intestinal stem cell quiescence. J Cell Biol, 215(3), 401-413. doi:10.1083/jcb.201604119

Zhang, Y., \& Kalderon, D. (2001). Hedgehog acts as a somatic stem cell factor in the Drosophila ovary. Nature, 410(6828), 599-604. doi:10.1038/35069099

Zielke, N., Korzelius, J., van Straaten, M., Bender, K., Schuhknecht, G. F. P., Dutta, D., . . . Edgar, B. A. (2014). Fly-FUCCI: A versatile tool for studying cell proliferation in complex tissues. Cell Rep, 7(2), 588-598. doi:10.1016/j.celrep.2014.03.020 